\title{
Quantum coherence and correlations of optical radiation by atomic ensembles interacting with a two-level atom in a microwave cavity
}

\author{
Ö. E. Müstecaplığ lu* \\ Department of Physics, Koç University, 34450 Sarlyer, Istanbul, Turkey and Institute of Quantum Electronics, ETH Zurich, \\ CH-8093 Zurich, Switzerland \\ (Received 27 July 2010; published 9 February 2011)
}

\begin{abstract}
We examine quantum statistics of optical photons emitted from atomic ensembles which are classically driven and simultaneously coupled to a two-level atom via microwave photon exchange. Quantum statistics and correlations are analyzed by calculating second-order coherence degree, von Neumann entropy, spin squeezing for multiparticle entanglement, as well as genuine two- and three-mode entanglement parameters for steady-state and nonequilibrium situations. Coherent transfer of population between the radiation modes and quantum-state mapping between the two-level atom and the optical modes are discussed. A potential experimental realization of the theoretical results in a superconducting coplanar waveguide resonator containing diamond crystals with nitrogen-vacancy color centers and a superconducting artificial two-level atom is discussed.
\end{abstract}

DOI: 10.1103/PhysRevA.83.023805

PACS number(s): 42.50.Pq, 76.30.Mi, 03.67.Lx

\section{INTRODUCTION}

Primary requirements of successful quantum information technology, in particular of large-scale distributed quantum networking, is to have efficient and reliable means of storage [1], processing [2], and dissemination [3] of quantum resources. These tasks can most likely be solved in different modules, designed and optimized for their ideal operation. The composite quantum information device would be then a hybrid structure of such subsystems [4]. As the individual modules can have different time and energy scales of operation, the fundamental challenge is to merge them together congruously. After the recent dramatic developments in hybrid systems of spin ensembles and superconducting cavities [5-7], an essential step toward distributed quantum information processing is to find means of coherent coupling of single microwave and optical photons [8].

In this article we consider an atomic three-level ensemble, with the so called $\Delta$-type level scheme $[9,10]$. We argue that such a system can be a promising setup for controllable exchange of quantum resources between energetically remote modules in a hybrid quantum information device. The basic idea is to exploit the presence of an extra energetic component in the $\Delta$ scheme to bridge the large energy gap between the other two components. In particular, we envision a typical case where one component is a two-level atom, corresponding to the stationary quantum hardware and quantum information unit (qubit). It is coupled to the $\Delta$ system by a cavity field in the microwave domain. The other component could be a remote quantum communication node such as a satellite or quantum repeater along an optical fiber so these require coupling to the $\Delta$ system in the optical domain. Hence, by choosing the extra available transition also in the optical domain, we can consolidate these two domains. The $\Delta$ system could play a double-faceted role. It can serve as the quantum memory in terms of the pure or, together with the two-level atom, hybrid spin ensemble qubits [11]. In addition, it becomes a mediator of communication between the stationary quantum hardware

\footnotetext{
*omustecap@ku.edu.tr
}

and the quantum communication channels for optical flying qubits interconnecting remote nodes of a quantum network.

Due to dipole selection rules, $\Delta$-type transitions can happen only in certain systems, either lacking, or with explicitly broken, inversion symmetry, such as artificial atoms [9,12], semiconductors [13-15], or chiral molecules [16,17]. Alternatively, one of the transitions can be of magnetic dipole or electric quadrupole type in the $\Delta$ scheme [18-20]. Such weak transitions are collectively enhanced for strong coupling due to the ensemble [11]. We specifically consider the latter case of magnetic dipole assisted $\Delta$ scheme, though our results would be valid in other cases, too.

Some quantum statistical properties of single isolated $\Delta$ type atomic ensembles have been explored recently [10,21]. Coupling of atomic ensembles, in particular, three-level $\Lambda$ type ensembles to a two-level atom has been discussed in the context of quantum-state mapping from quantum memories to charge qubit [22] in transmission line resonators [23-26]. In this article we examine quantum coherence and correlations of the optical photons emitted by a $\Delta$-type ensemble coupled to a two-level atom in a microwave cavity. We also investigate the case of two $\Delta$-type ensembles. Equilibrium and nonequilibrium situations are separately examined. Conditions of photon antibunching, particle entanglement $[27,28]$, genuine bipartite and tripartite mode entanglement, $W$ state [29] are revealed. Furthermore, quantum-state mapping between the two-level atom and optical modes, as well as coherent transfer of population between the radiation modes are found.

This article is organized as follows. In Sec. II we describe the model system and by eliminating the collective atomic states, we derive the effective Hamiltonians for two-level atom and radiation modes. In Sec. III, we present our results and discussions for the single and two-ensemble cases in the steady-state and nonequilibrium situations. In Sec. IV, a potential experimental system for physical implementation of our results is described. Specifically, we consider an extension of recently realized nitrogen-vacancy $(\mathrm{N}-\mathrm{V})$ centers in diamond crystals strongly coupled to superconducting coplanar waveguide resonators (CPWG) [5]. Similar electron spin ensemble to on-chip superconducting cavity couplings, specifically coupling of $\mathrm{Cr}^{3+}$ spins in ruby and $\mathrm{N}$ substitution (P1) 


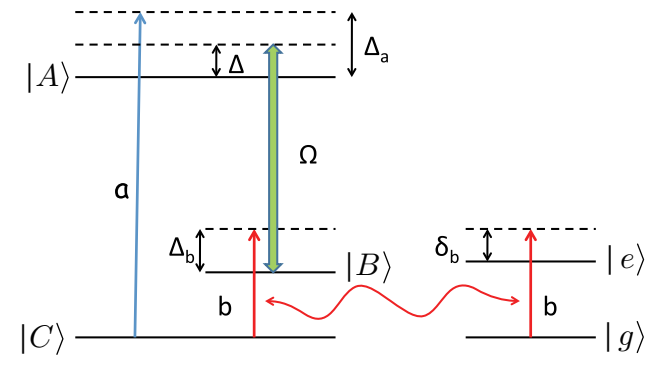

FIG. 1. (Color online) Identical three-level atoms in the ensemble interact with two quantized (indicated by $a$ and $b$ ) and one classical (indicated by $\Omega$ ) electromagnetic fields in $\Delta$ scheme. The microwave photons further couple the atomic ensemble to a single two-level atom. The classical drives on C-A transition and on the cavity field are not shown.

centers in diamond, are also observed in parallel efforts [6]. Finally, in Sec. V, we conclude.

\section{II. $\triangle$-TYPE ATOMIC ENSEMBLE COUPLED TO A TWO-LEVEL ATOM}

\section{A. Model Hamiltonian}

We consider a collection of $N$ identical three-level atoms interacting with three electromagnetic fields in a $\Delta$-type transition scheme delineated in Fig. 1. The atomic ensemble is further coupled to a two-level atom through microwave photon exchange. The upper and lower levels of the two-level atom are respectively denoted by $|e\rangle$ and $|g\rangle$. The lowest level $|C\rangle$ is coupled to the intermediate level $|B\rangle$ by a magnetic dipole transition of rate $g_{b}$, while the transitions from the highest level $|A\rangle$ to the lower doublet $|B\rangle$ and $|C\rangle$ are optical electric-dipole transitions of rates $\Omega$ and $g_{a}$, respectively. We further suppose that optical fields are traveling waves while the microwave field is a standing wave of a cavity that contains the two-level atom and the atomic ensemble.

Our model can be conceived as a modest generalization of the case of a $\Delta$-type atomic ensemble, resonantly coupled to the optical fields, which is studied for the bipartite entanglement of the quantized fields [10]. On the other hand, such a seemingly innocent addition of two-level atoms generalizes the system from two interacting linear oscillators to the case of a linear oscillator coupled to a nonlinear oscillator and, as such, can cause remarkable changes in the quantum statistical properties of the fields. In comparison to $\Lambda$-type ensemble coupled to two-level atom [30], $\Delta$-type scheme allows for effective interaction between $a$ and $b$ modes. This effective interaction leads to correlations between the photon modes, so-called mode entanglement, which can be characterized by the entanglement entropy.

On the other hand, pairwise or multiparticle entanglement requires nonlinear interactions and cannot be obtained by simple coupled linear oscillators. Inclusion of two-level atoms brings back the missing nonlinearity and anharmonicity to the $\Delta$-type atomic ensemble. In such a system we can enjoy the benefits of collective enhancement of coupling coefficient by the ensemble together with strictly nonlinear effects such as photon blockade or antibunching or multiparticle entanglement which is characterized by the spin squeezing [27,28].

Furthermore, the coupled linear oscillator models can be viewed as nonlocal or long-range interactions which may arise as hopping or tunneling couplings. Introduction of the twolevel atom brings the local or short-range interaction into the picture. Competing effects of localization and delocalization can be examined in terms of mode and particle entanglement when the two-level atom is present. Finally, from a practical point of view, the model has all the ingredients (memory, processor, bus and flying qubits) in the microwave and optical frequency domains. It allows for a compact examination of the coherent coupling of these modules for distributed quantum information purposes.

In addition, we take into account additional external drives and consider the system as open with all the decay channels due to cavity losses or spontaneous emission decays, which causes subtle effects in particular in the steady state. In addition to that, we also examine the case of two distant ensembles to analyze genuine three-mode entanglement possibilities, in particular to look for $W$-state generation among microwave and optical photons. The question of how to realize these models in a practical and recent system will be subject to Sec. IV while the numerical simulations will use the corresponding, experimentally available, set of parameters.

The system can be described by a model Hamiltonian $H=$ $H_{0}+H_{1}+H_{p}$, where (in units of $\hbar$ )

$$
\begin{aligned}
H_{0}= & \sum_{\vec{k}} \omega_{a \vec{k}} a_{\vec{k}}^{\dagger} a_{\vec{k}}+\omega_{b} b^{\dagger} b+\frac{\omega_{0}}{2} \sigma_{z}+\sum_{x j} \omega_{x} R_{x x}^{(j)}, \\
H_{1}= & g \sigma b^{\dagger}+\Omega \sum_{j} R_{A B}^{(j)} e^{i\left(\vec{k}_{d} \cdot \vec{r}_{j}-\omega_{d} t\right)} \\
& +\sum_{j, \vec{k}} g_{a \vec{k}} e^{i \vec{k} \cdot \vec{r}_{j}} R_{A C}^{(j)} a_{\vec{k}}+g_{b} \sum_{j} R_{B C}^{(j)} b+\text { H.c. }, \\
H_{p}= & \Omega_{A} \sum_{j} R_{A C}^{(j)} e^{i\left(\vec{k}_{A} \cdot \vec{r}_{j}-\tilde{\omega}_{A} t\right)}+E_{b} e^{-i \tilde{\omega}_{b} t} b+\text { H.c. }
\end{aligned}
$$

Here, Pauli spin-1/2 operators for the two-level atom $\sigma=$ $|g\rangle\langle e|$ and $\sigma_{z}=|e\rangle\langle e|-| g\rangle\langle g|$ respectively stand for the lowering and the inversion operators. The labels $x=A, B$ and $j=1, \ldots, N$ denote the corresponding energy levels and the index of the atom in the ensemble. The frequencies $\omega_{a \vec{k}}, \omega_{b}, \omega_{A}, \omega_{B}, \omega_{0}$ are respectively the frequencies of the electromagnetic fields and atomic transitions relative to the lowest level $|C\rangle$. Probe field frequencies are denoted by $\tilde{\omega}_{A}$ and $\tilde{\omega}_{b}$. Effective drive strength of the microwave cavity and the Rabi frequency of the drive for the $|C\rangle$ to $|A\rangle$ transition are represented by $E_{b}$ and $\Omega_{A}$, respectively. Population and transition operators of single atoms in the ensemble are respectively represented by $R_{x x}^{(j)}=|x\rangle_{j j}\langle x|$ and $R_{x y}^{(j)}=$ $|x\rangle_{j j}\langle y|, x \neq y$ with $x, y=A, B, C$. Optical and microwave photon annihilation (creation) operators denoted by $a_{\vec{k}}, b$ $\left(a_{\vec{k}}^{\dagger}, b^{\dagger}\right)$, respectively.

Cavity photon operator $b$ implicitly depends on the wave number of the cavity mode. The coupling coefficient $g_{b}$ is assumed to be spatially homogeneous over the ensemble so a mode-matching condition [10] only for the wave vectors of the optical fields can be imposed such that $\vec{k}=\vec{k}_{d}=\vec{k}_{A}$. 
Quantized optical field can be treated as single mode and the coupling to the reservoir of the other modes can be treated as a decay channel in the master equation for the model system. More detailed conditions on three-dimensional analysis of mode matching between free space light and the atomic ensemble will be ignored for simplicity [31]. Suppressing the constant global phase factor by [32] $|A\rangle \rightarrow$ $\exp \left(i \vec{k}_{d} \cdot \vec{r}_{j}\right)|A\rangle$, we drop the $\vec{k}$ index from our notations and further considerations.

There would be additional Langevin noise terms in the Hamiltonian, corresponding to the coupling to the continuum of other modes. These will be taken into account effectively in the master equation formalism and shall not be shown here.

\section{B. Quasispin wave picture of the model system}

It is illuminating to portray the model system in terms of collective excitation operators [33] $A=X_{C A} / \sqrt{N}, B=$ $X_{C B} / \sqrt{N}, T=X_{B A}$, where $X_{x y}=\sum_{j=1}^{N} R_{x y}$, is analogous to the Hubbard operators [34] in strongly correlated systems. In the ensemble, the set of Hubbard operators represent the SU(3) symmetry of a spin-1 system. They can be associated with the Gell-Mann fundamental representation of the SU(3) Lie algebra by choosing $U=X_{C A}, V=X_{B C}$ and $T=X_{B A}$, so $U, V$, and $T$ form the three spin- $1 / 2$ subgroups. Each obeys the $\mathrm{SU}(2)$ algebra with $\left[L, L^{\dagger}\right]=-2 L_{3}, L=U, V, T$. They are interdependent by the commutations such as $\left[V^{\dagger}, U^{\dagger}\right]=-T^{\dagger}$. Analogous to excitons and magnons, SU(2) algebras of $U$ and V subspins can be contracted to Weyl-Heisenberg algebra $h_{2}$ to associate collective excitations with quasi-spin-wave quanta [33]. The system space becomes a semidirect product Lie algebra, $\mathrm{SU}(2) \bar{\otimes} h_{2}$. In the limit of low excitations to $|A\rangle$ and $|B\rangle$, relative to $N, V_{3} \approx N / 2$, and $U_{3} \approx-N / 2$. With the contraction parameter $1 / \sqrt{N}$, the operators $A=U / \sqrt{N}$ and $B=V^{\dagger} / \sqrt{N}$ become approximately bosonic. They become independent, as the isospin subgroup is weakly populated. The isospin is consistently represented by $T=B^{\dagger} A$.

Denoting the phases of the $g, g_{a}, g_{b}, \Omega, \Omega_{A}, E_{b}$ by $\theta_{g}, \theta_{a}$, $\theta_{b}, \theta_{\Omega}, \theta_{A}, \tilde{\theta}_{b}$, respectively, they can be suppressed by $b \rightarrow$ $b \exp \left(i \tilde{\theta}_{b}\right), \sigma \rightarrow \sigma \exp \left[i\left(\theta_{g}-\tilde{\theta}_{b}\right)\right], B \rightarrow B \exp \left[-i\left(\theta_{b}+\tilde{\theta}_{b}\right)\right]$, $a \rightarrow a \exp \left[i\left(\theta_{a}+\theta_{A}\right)\right]$, and $A \rightarrow A \exp \left(-i \theta_{A}\right)$. Employing a unitary transformation by $U=\exp \left(-i \omega_{d} t\right)$ with $H \rightarrow U^{\dagger} H U-i U^{\dagger} \partial_{t} U$, the Hamiltonian becomes

$$
\begin{gathered}
H_{0}=\sum_{x=a, A} \varpi_{x} x^{\dagger} x+\omega_{b} b^{\dagger} b+\omega_{B} B^{\dagger} B+\frac{\omega_{0}}{2} \sigma_{z}, \\
H_{1}=g \sigma b^{\dagger}+g_{a} A^{\dagger} a+g_{b} B^{\dagger} b+\Omega e^{i \phi} A^{\dagger} B+\text { H.c., } \\
H_{p}=\Omega_{A} e^{-i \tilde{\varpi}_{A} t} A^{\dagger}+E_{b} e^{-i \tilde{\omega}_{b} t} b+\text { H.c. }
\end{gathered}
$$

where the coupling coefficients $g_{a, b} \rightarrow \sqrt{N} g_{a, b}$ are collectively enhanced; $\phi=\theta_{\Omega}+\theta_{A}-\theta_{b}-\tilde{\theta}_{b}, \varpi_{a, A}=\omega_{a, A}-\omega_{d}$, and $\tilde{\omega}_{A}=\tilde{\omega}_{A}-\omega_{d}$. Even though it can play a curious role in quantum correlations [10], for simplicity we take $\phi=0$. We further assume a three-photon resonance condition $\tilde{\varpi}_{A}=$ $\tilde{\omega}_{A}-\omega_{d}=\tilde{\omega}_{b}$ for the classical driving fields.

\section{Effective Hamiltonian for the model system}

A $\Delta$-type ensemble can induce an effective photon-photon interaction mediated by the quasi-spin-wave background [10].
In order to show that explicitly, let us introduce the normal modes $p_{ \pm}=u_{ \pm} A+v_{ \pm} B$ by the Bogoliubov transformation [35] with the relations $u_{-}=-v_{+}$and $v_{-}=u_{+}$, where $u_{ \pm}=$ $\sqrt{\left[\eta \pm\left(\varpi_{A}-\omega_{B}\right)\right] / 2 \eta}$ and $\eta=\sqrt{\left(\varpi_{A}-\omega_{B}\right)^{2}+4 \Omega^{2}}$.

The model Hamiltonian can be rewritten as $H=H_{0}+$ $H_{1}+H_{p}$, where

$$
\begin{gathered}
H_{0}=\varpi_{a} a^{\dagger} a+\omega_{b} b^{\dagger} b+\sum_{\lambda= \pm} \Omega_{\lambda} p_{\lambda}^{\dagger} p_{\lambda}+\frac{\omega_{0}}{2} \sigma_{z}, \\
H_{1}=g \sigma b^{\dagger}+\sum_{\lambda= \pm} p_{\lambda}^{\dagger}\left(g_{a \lambda} a+g_{b \lambda} b\right)+\text { H.c. } \\
H_{p}=\sum_{\lambda= \pm} \Omega_{A \lambda} e^{-i \tilde{\varpi}_{A} t} p_{\lambda}+E_{b} e^{-i \tilde{\omega}_{b} t} b+\text { H.c. }
\end{gathered}
$$

Here $\Omega_{ \pm}=\left(\varpi_{A}+\omega_{B} \pm \eta\right) / 2, g_{a \lambda}=g_{a} u_{\lambda}, g_{b \lambda}=g_{b} v_{\lambda}$, and $\Omega_{A \lambda}=\Omega_{A} u_{\lambda}$.

Effective coupling between the electromagnetic modes can be obtained by a unitary transformation to eliminate the interactions between the electromagnetic and quasi-spin-wave modes. We introduce an anti-Hermitian operator $S$

$$
S=\sum_{\lambda= \pm}\left(x_{\lambda} a+y_{\lambda} b\right) p_{\lambda}^{\dagger}-\text { H.c. }
$$

where the unknown coefficients $x_{\lambda}$ and $y_{\lambda}$ are to be determined by the Fröchlich-Nakajima canonical transformation condition [36-38]

$$
\left[H_{0}, S\right]=-\sum_{\lambda= \pm} p_{\lambda}^{\dagger}\left(g_{a \lambda} a+g_{b \lambda} b\right)+\text { H.c. }
$$

which yields $x_{\lambda}=g_{a \lambda} /\left(\varpi_{a}-\Omega_{\lambda}\right)$ and $y_{\lambda}=g_{b \lambda} /\left(\omega_{b}-\Omega_{\lambda}\right)$.

Assuming $\Omega \gg g_{a}, g_{b}$, an effective Hamiltonian $H_{\text {eff }}=$ $\exp (-S) H \exp (S)$ can be written up to the second order in $x_{\lambda}, y_{\lambda}$,

$$
\begin{aligned}
H_{\mathrm{eff}}= & \varpi_{a}^{\prime} a^{\dagger} a+\omega_{b}^{\prime} b^{\dagger} b+\frac{\omega_{0}}{2} \sigma_{z}+g\left(\sigma b^{\dagger}+\text { H.c. }\right) \\
& +\left(E_{b}^{\prime} e^{-i \tilde{\omega}_{b} t} b+E_{a} e^{-i \tilde{\omega}_{A} t} a^{\dagger}-J a^{\dagger} b+\text { H.c. }\right) \\
& +\sum_{\lambda} \Omega_{\lambda}^{\prime} p_{\lambda}^{\dagger} p_{\lambda}+\sum_{\lambda \neq \mu} Q_{\lambda \mu}\left(p_{\lambda}^{\dagger} p_{\mu}+\text { H.c. }\right) \\
& +\sum_{\lambda}\left(G_{\lambda} \sigma p_{\lambda}^{\dagger}+E_{p \lambda} e^{-i \tilde{\omega}_{b} t} p_{\lambda}^{\dagger}+\text { H.c. }\right)
\end{aligned}
$$

with $\lambda, \mu= \pm$. The coefficients in $H_{\text {eff }}$ are listed in the Appendix A. The indirect coupling between the quasi-spinwave modes and the electromagnetic modes is through their common interaction with the two-level atom. In the large $\Omega$ limit, the quasispin waves are strongly off-resonant with the two-level atom and practically uncoupled. More formally, in the dispersive regime, $\Omega-\omega_{0} \sim \Omega \gg g$, employing another Fröchlich transformation, such an interaction can be reduced to a Stark shift of a two-level atom resonance proportional to the population of the quasispin normal modes that can be neglected for weak excitations. Influence of such sequential canonical transformation on radiation modes would be negligible provided that $\Omega \gg g_{a} \gg g_{b}, g$. In addition, the effective weak drive on the two-level atom would be negligible. The effective Hamiltonian, keeping the leading terms only, is 
reduced to

$$
\begin{aligned}
H_{\mathrm{eff}}= & \left(\varpi_{a}-\tilde{\omega}_{b}\right) a^{\dagger} a+\left(\omega_{b}-\tilde{\omega}_{b}\right) b^{\dagger} b+\frac{\omega_{0}-\tilde{\omega}_{b}}{2} \sigma_{z} \\
& +g\left(\sigma b^{\dagger}+\text { H.c. }\right)-J\left(a^{\dagger} b+\text { H.c. }\right) \\
& +E_{a}\left(a^{\dagger}+a\right)+E_{b}\left(b^{\dagger}+b\right) .
\end{aligned}
$$

Here, three-photon resonance condition for the driving classical fields is employed and the Hamiltonian is written in the frame rotating at $\tilde{\omega}_{b}$. We further suppose that three-photon resonance holds for the radiation fields such that $\omega_{a}=$ $\omega_{d}+\omega_{b}$, then $\varpi_{a}-\tilde{\omega}_{b}=\omega_{b}-\tilde{\omega}_{b} \equiv \delta$ is defined. We find $\omega_{0}-\tilde{\omega}_{b}=\delta-\delta_{b}$. According to Fig. $1, \delta_{b}=\omega_{b}-\omega_{0}, \Delta=$ $\omega_{d}-\omega_{A B}, \Delta_{a}=\omega_{a}-\omega_{A}, \Delta_{b}=\omega_{b}-\omega_{B}$, in terms of which we express the three-photon resonance condition $\Delta_{a}-\Delta-$ $\Delta_{b}=0$.

The Hamiltonian of Eq. (13) has a zero-eigenvalue solution in the single excitation manifold, or the so-called dark state, which is given by [30]

$$
|\mathrm{DS}\rangle=(\cos \alpha \sigma-\sin \alpha a)^{\dagger}|\mathrm{vac}\rangle,
$$

with $\alpha=-\arctan (g / J)$. Presence of such a dynamically fixed state has been exploited to propose an adiabatic transfer protocol to map the quantum information stored in a molecular $\Lambda$ type ensemble to a charge qubit of two-level atom and vice versa. Our treatment of cyclic ensembles reveals a complement to this protocol by allowing quantum information transfer from a two-level atom stationary qubit to an optical flying qubit. A state $(x|g\rangle+y|e\rangle)|00\rangle_{a b}$ can be adiabatically transferred into $\left(x|0\rangle_{a}+y|1\rangle_{a}\right)|g\rangle|0\rangle_{b}$. The decoherence channels due to two-level atom and the microwave cavity are suppressed. The effective loss channel of the optical photon, transferred from the spontaneous emission of the level $|A\rangle$ during the Fröchlich transform, is compensated with the effective drive $E_{a}$. Taking into account time-dependent interaction coefficients and timedependent canonical transformations, an optimal fidelity of the state transfer can be characterized.

Alternative to the derivation based on sequential Fröchlich transformations as presented here, it is possible to get the effective Hamiltonian with a single-step Fröchlich transformation or by reducing a general four-color $(a, b, A, B)$ polariton to a two-color $(a, b)$ one [10] for strong drive $\Omega$ conditions. It is straightforward to generalize this model by either of these methods to the case of a pair of cyclic atomic ensembles in the microwave cavity, coupled to the same two-level atom. We find

$$
\begin{aligned}
H_{\mathrm{eff}}= & \sum_{j=1,2} \varpi_{a j}^{\prime} a_{j}^{\dagger} a_{j}+\omega_{b}^{\prime} b^{\dagger} b+\frac{\omega_{0}}{2} \sigma_{z} \\
& +g\left(\sigma b^{\dagger}+\text { H.c. }\right)-\sum_{j=1,2} J_{j}\left(a_{j}^{\dagger} b+\text { H.c. }\right) \\
& +\sum_{j=1,2} E_{j}\left(a_{j}^{\dagger}+a_{j}\right)+E_{b}\left(b^{\dagger}+b\right)
\end{aligned}
$$

for identical symmetric ensembles, $\varpi_{a j}=\varpi_{a}, E_{j}=E_{a}$, and $J_{j}=J$.

The single ensemble effective model reduces to the JaynesCummings (JC) model for the optical mode in the dispersive limit of the microwave cavity. The two-ensemble model becomes a two-site Bose-Hubbard $(\mathrm{BH})$ model, with pure and cross-Kerr interactions of the type $\left(a_{i}^{\dagger} a_{i}\right)^{2}, i=1,2$ and $a_{1}^{\dagger} a_{1} a_{2}^{\dagger} a_{2}$. A common proposal to realize a BH dimer is to use JC-BH systems of tunnel coupled nonlinear cavities, where the nonlinearity is provided by a two-level atom in each of them $[39,40]$. In contrast to such direct methods to realize atom-optical analogs of Josephson networks, here there is only one two-level atom providing shared nonlinearity by each optical mode, which simplifies architecture of identical homogeneous coupled subsystems.

The BH dimer can be described as a pseudospin system with quadratic nonlinearities using pseudospin Schwinger operators $S_{+}=a_{1}^{\dagger} a_{2}, S_{z}=\left(a_{1}^{\dagger} a_{1}-a_{2}^{\dagger} a_{2}\right) / 2$. When sufficiently strong nonlinearity present, it leads to multiparticle entanglement between the distant optical modes via the so-called axis twisting spin-squeezing route [27,28]. The question of whether multiparticle entanglement can still be found in the nondispersive regime is not as obvious to answer due to lack of immediate mapping onto a spin model. We shall explore that case numerically in the following section, together with other types of quantum correlations as well as with quantum statistical properties of radiation modes in the effective models.

Generalization of these models beyond two ensembles is, in principle, possible. However, the quasi-spin-wave-mediated effective tunnel coupling here is long range beyond nearest neighbors and hence a direct relation to simple Bose-Hubbard models is not immediate.

\section{RESULTS AND DISCUSSIONS}

Assuming the effective model remains Markovian and Born-Markov approximation is applicable, dynamics can be investigated by a quantum master equation

$\dot{\rho}=-i[H, \rho]+\sum_{x} \kappa_{x} \mathcal{D}[x] \rho+\gamma \mathcal{D}[\sigma] \rho+\frac{\gamma_{\phi}}{2} \mathcal{D}\left[\sigma_{z}\right] \rho$.

Here $\kappa_{x}$ is the photon loss rate of the mode $x=a, b$ and $\gamma_{1}$ and $\gamma_{\phi}$ are respectively the relaxation and pure dephasing rates of the two-level atom. Dissipation terms $\mathcal{D}[x] \rho=$ $\left(2 x \rho x^{\dagger}-x^{\dagger} x \rho-\rho x^{\dagger} x\right) / 2$ are the Liouvillian superoperators for damping in Lindblad form.

Fröchlich transformations used in the derivations of the effective models influence the decay rates. In the leading order, we estimate $\kappa_{a} \sim \gamma_{A}\left(g_{a} / \Omega\right)^{2}$, where $\gamma_{A}$ is the excited state decay rate of the $\Delta$-type atom. We choose $g_{a} / \Omega=0.1$ so $\kappa_{a}=0.01 \gamma_{A}$. We assume $\kappa_{a}=\kappa_{b}$. In addition, we suppose $E_{a}=E_{b}=0.1 \kappa_{b}$, which corresponds to the weak driving condition for which the empty cavity photon number is 0.01 on resonance. Such strict equalities are to reduce the number of independent parameters in the simulations but not crucial in practice. It is sufficient to arrange field-atom detunings and the driving field strengths. (The formulas in the Appendix A can be referred) to ensure sufficient effective drive $E_{a}$ can be translated from $A$ to $a$ so that the optical radiation can survive in the steady state against the decay channels.

We numerically solve the master equation using the "Quantum Optics Toolbox" [41] and determine the steady-state and dynamical behavior of the system. Our typical results will be reported below for the cases of single and two-atomic ensembles separately. For all cases we fix $J / 2 \pi=1 \mathrm{MHz}$. 
(a)
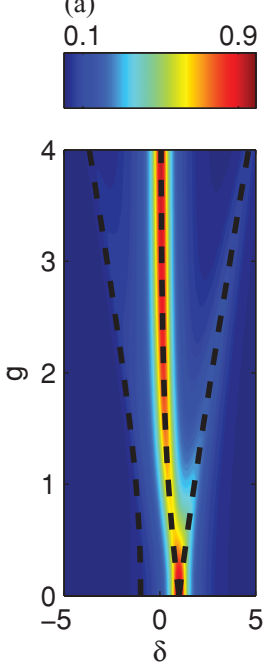

(b) 0.1
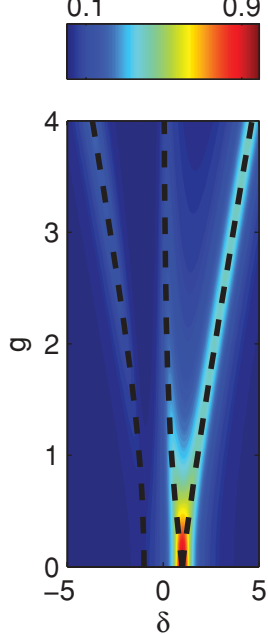

(c)
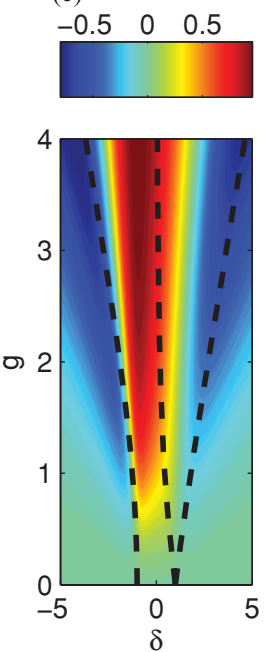

FIG. 2. (Color online) Steady-state populations (a) $n_{a}$ and (b) $n_{b}$ and (c) their relative fractional population $n_{a b}=\left(n_{a}-n_{b}\right) /\left(n_{a}+n_{b}\right)$ of the optical and microwave modes at $J / 2 \pi=1 \mathrm{MHz}, \delta_{b}=J$, $\kappa_{b}=\kappa_{a} /=(2 \pi) 0.4 \mathrm{MHz}, \gamma_{1} / 2 \pi=0.02 \mathrm{MHz}, \gamma_{\phi} / 2 \pi=0.3 \mathrm{MHz}$. Fock spaces for the radiation modes are taken to be four dimensional. Dashed lines indicate the probe resonances with the single excitation manifold. $g / 2 \pi$ and $\delta / 2 \pi$ are in megahertz.

\section{A. Single atomic ensemble}

\section{Steady-state coherence and correlations}

Taking $\delta_{b}=J, \quad \kappa_{b}=\kappa_{a}=(2 \pi) 0.4 \mathrm{MHz}, \quad \gamma_{1} / 2 \pi=$ $0.02 \mathrm{MHz}, \gamma_{\phi} / 2 \pi=0.3 \mathrm{MHz}$, the steady-state solution of the master equation is determined, in a truncated Fock space of three photons in each mode, for a range of $g / 2 \pi \in\{0,4\}$ $\mathrm{MHz}$ and $\delta / 2 \pi \in\{-5,5\} \mathrm{MHz}$.

Populations of the radiation modes are shown in Fig. 2 in the $(\delta, g)$ phase space. Dashed lines indicate the probe resonances with the single excitation manifold. The relatively low transmission along a particular resonance is due to our choice of equal drive strengths. For a simple illustration, let us consider $\delta_{b}=0$ for which

$$
\begin{gathered}
q_{D}=\frac{-g}{K} a-\frac{J}{K} \sigma, \\
q_{ \pm}=\frac{1}{\sqrt{2} K}(-J a \pm K b+g \sigma),
\end{gathered}
$$

are the dressed atom-polariton operators [42] of the single excitation manifold. For our choice of $\delta_{b}=0, q_{D}^{\dagger}|\mathrm{vac}\rangle$ is the dark state of the manifold. The states generated by the $q_{ \pm}^{\dagger}$ are with $\pm K$ energies, where $K=\sqrt{g^{2}+J^{2}}$. For $g<J$ we find $a=\left(q_{+}+q_{-}\right) / \sqrt{2}$ and $b=\left(q_{+}+q_{-}\right) / \sqrt{2}$ so the combined drive terms for the $a$ and $b$ modes becomes $\sqrt{2} E_{a}\left(q_{+}+q_{+}^{\dagger}\right)$ if $E_{a}=E_{b}$. In that case neither $q_{-}$nor $q_{D}$ are driven and hence they cannot survive to steady state. Figure 2 confirms this argument as it exhibits that only the bright polariton branch is the upper one, while the lower two remains in the dark. With the increase of $g, a$ mode drives mainly the $q_{D}$ polariton. This is seen in Fig. 2 as the bright middle polariton branch. The upper branch of $q_{+}$is more strongly driven relative to $q_{-}$and correspondingly associated with the bright $b$ line in Fig. 2(b).

One can selectively populate different polariton branches; for example, taking $E_{b}=-E_{a}$ yields bright $b$ mode along the lower polariton branch.

Figure 2(c) is the behavior of population fraction defined to be $w=\left(n_{a}-n_{b}\right) /\left(n_{a}+n_{b}\right)$, with $n_{x}=\left\langle x^{\dagger} x\right\rangle, x=a, b$. As long as $g<J$ there is no distinct radiation phases in the $(\delta, g)$ space. When $g>J$, however, radiation in the system is dominated by the microwave mode along the upper and lower polariton branches. In between, there is a wedgelike region about the middle polariton branch, where the radiation is dominated by the optical mode. The main effect of nonzero $\delta_{b}$ would be to change the slopes of the probe resonance lines, and as such the widths of the optical and microwave phases, but the above descriptions would be valid.

We characterize quantum statistical coherence properties of the radiation modes in the steady state in terms of the second-order coherence function at zero time delay,

$$
g^{2}(0)=\frac{\left\langle a^{\dagger} a^{\dagger} a a\right\rangle}{\left\langle a^{\dagger} a\right\rangle^{2}},
$$

the von Neumann entanglement entropy [2],

$$
S=-\operatorname{Tr}\left(\rho_{a} \log _{2} \rho_{a}\right),
$$

and the genuine two-mode entanglement parameter [43],

$$
\lambda_{a b}=\left|\left\langle a^{\dagger} b\right\rangle\right|^{2}-\left\langle a^{\dagger} a b^{\dagger} b\right\rangle .
$$

Here $\rho_{a}=\operatorname{Tr}_{b}\left[\operatorname{Tr}_{\sigma}\left(\rho_{s s}\right)\right]$ is the reduced density matrix of optical mode $a$ evaluated by tracing out atomic $(\sigma)$ and microwave $(b)$ degrees of freedom. $S$ is an entanglement measure for pure bipartite states. As we have three subsystems, we can interpret it as a measure of impurity in the steady state, analogous to linear entropy. On the other hand, we find that the two-level atom is weakly excited everywhere in the $\delta, g$ phase space for the steady state. Thus, $S$ bears the signs of profound quantum correlations between the radiation modes, especially in the $g<J$ regime, where it should be a reliable measure of mode entanglement. A more clear signature of genuine two-mode entanglement can be found when $\lambda_{a b}>0$.

Mode entanglement is due to correlations in the occupation number space in the second quantization framework. In the first quantization or in the particle picture, scattering of particles can lead to multiparticle entanglement. Though we do not have such direct particle interactions for photons, effective interactions that lead to photon blockade effect $[44,45]$ can yield similar multiparticle entanglement of photons. We want to explore if increased particle correlations among the photons can be found with the increasing anharmonicity parameter $g$ in regimes of sub-Poisson statistics. To characterize particle entanglement, we use a spin-squeezing parameter [27,28], generalized to the case of dissipation [46], defined to be

$$
\xi_{x}^{2}=\frac{\langle N\rangle\left[\Delta J_{x}\right]^{2}}{\left\langle J_{y}\right\rangle^{2}+\left\langle J_{z}\right\rangle^{2}} .
$$

When $\xi_{x}^{2}<1$ the state is said to be particle entangled, so it has nonseparable density matrix in the first quantization. Here the pseudospin operators $J_{x, y, z}$ are the usual Schwinger 
(a)

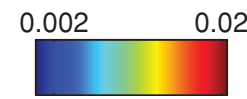

(b)
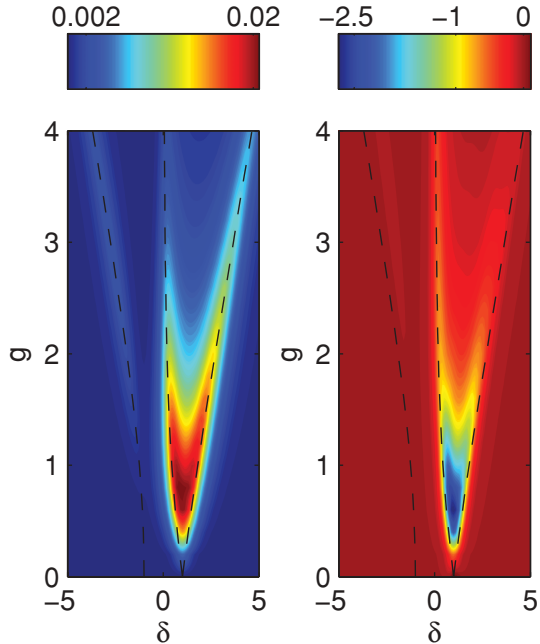

(c)
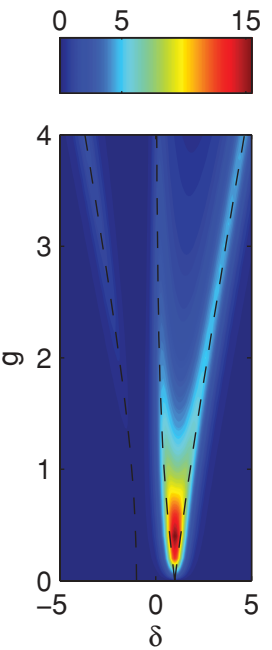

FIG. 3. (Color online) Same as shown in Fig. 2 but for (a) the von Neumann entanglement entropy $S$, (b) genuine two-mode entanglement parameter $\lambda_{a b}$, and (c) the excited-state population $\sigma_{e e}=\sigma^{\dagger} \sigma . \lambda_{a b}$ is multiplied by $10^{5}$ and $\sigma_{e e}$ is multiplied by $10^{3}$ for visibility. Dashed lines indicate the probe resonances with the single excitation manifold. $g / 2 \pi$ and $\delta / 2 \pi$ are in megahertz.

representation for the two bosonic modes $a$ and $b$,

$$
\begin{aligned}
J_{x} & =\frac{1}{2}\left(a^{\dagger} b+a b^{\dagger}\right), \\
J_{y} & =\frac{1}{2}\left(a^{\dagger} b-a b^{\dagger}\right), \\
J_{z} & =\frac{1}{2}\left(a^{\dagger} a-b^{\dagger} b\right) .
\end{aligned}
$$

$\langle N\rangle=\left\langle a^{\dagger} a+b^{\dagger} b\right\rangle$ is the total number operator subject to losses. The squeezing is in general defined for an arbitrary axis, which makes it possible to optimize. However, it is more practical to examine a particular axis in the laboratory frame, which we choose to be the $x$ axis.

According to Fig. 3, $S$ is strongest about $M_{0}=(g, \delta) \sim$ $(J / 2, J)$. For $g>J$, it decreases but occurs predominantly along the polariton branches. The decrease with $g$ is due to reduction of the Hilbert space dimensionality. All three subsystems, two-level atoms and the radiation modes, are excited about the $M_{0}$ point, which can be seen in Figs. 23. The three-component atom-cavity "molecular" structure reduces to two-component forms as $g$ increases. The middle and right polariton branches become dominated by the $a$ and $b$ modes, respectively. Two-level atom excitation is mainly along the right branch. The middle figure indicates that genuine two-mode correlations are weakest about $M_{0}$ and stronger along the middle branch. The opposite behavior of $\lambda_{a b}$ and $S$ confirms the strong influence of the atomic component on $S$. $\lambda_{a b}<0$ reflects that $a-b$ correlations are atom mediated rather than genuine. Though not visible in the scale of the figure, $\lambda_{a b}>0$ is found in the vicinity of $(\delta=1, g=0)$ point, for which decay channels of the two-level atom is turned off and true two-mode entanglement is survived, though barely, in the steady state.

The second-order coherence functions for the modes $a, b$ together with the spin-squeezing parameter of multipartite entanglements are shown in Fig. 4. We plot $-\log _{10}\left[g_{a}^{2}(0)\right]$,



(b)
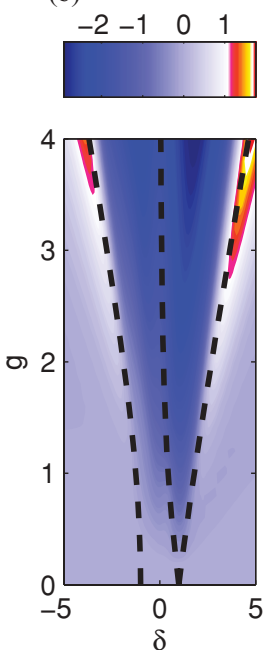

(c)


FIG. 4. (Color online) Same as shown in Fig. 2 but for (a) secondorder coherence functions $g_{a}^{2}(0)$, (b) $g_{b}^{2}(0)$, and (c) the spin-squeezing parameter $\xi_{x}^{2}$. These are plotted as $-\log _{10}\left[g_{a}^{2}(0)\right],-\log _{10}\left[g_{b}^{2}(0)\right]$, and $-\log _{10}\left(\xi_{x}^{2}\right) . g / 2 \pi$ and $\delta / 2 \pi$ are in megahertz.

$-\log _{10}\left[g_{b}^{2}(0)\right]$, and $-\log _{10}\left(\xi_{x}^{2}\right)$ so their positive values respectively mean sub-Poisson statistics for modes $a, b$ and particle entanglement of optical and microwave photons.

The behavior of the $g_{b}^{2}(0)$ for $g>J$ is typical in the JC model for $g<J, g_{b}^{2}(0) \sim g_{a}^{2}(0) \lesssim 0$. The equivalence of the $g^{2}(t)$ to Mandel $Q$ parameter [47] associates antibunched photons with $g^{2}<0$ with sub-Poissonian statistics. The only significantly populated antibunching region for mode $a$ is about $\delta \sim-0.5$, at the beginning of the optical domain. The other two antibunching regions of the optical mode are along the microwave domains and hence relatively weakly populated. The coherence properties of the microwave photon are transferred to the optical one via their common interaction with the two-level atoms. Collective enhancement of the interaction coefficients induces the bosonic (linear oscillator) character to the $\Delta$-type ensemble, which is harmful for sub-Poisson photon statistics. By addition of the two-level atom, the dressed system regains its lost anharmonic character. Enhancement of both $J$ and $g$ optimizes the antibunching of the optical photons. Despite relatively small coupling constants, the minimum $g_{a}^{2}(0) \sim 0.8$ and $g_{b}^{2}(0) \sim 0.1$ are within the same range with two-level nonlinear oscillators. A promising route to enhance these numbers could be to consider a more efficient source of anharmonicity, such as the $N$-type four-level system [48].

Nonlinearity of the JCM is associated with the anharmonic spacing of the energy levels. One prominent effect of anharmonic level spacing is photon blockade [44], which is the suppression of the sequential absorption of two photons into the system. In our case the anharmonicity influences the normal modes of the coupled $a, b$ modes similar to a case of photon blockade in a two-mode cavity. The degree of second-order coherence is equivalent to the Mandl $Q$ parameter which is a relative measure of number fluctuations. Photon blockade is associated with sub-Poisson statistics of the antibunched radiation. Since the normal mode transformation is a rotation 
in the mode space, the sub-Poisson statistics that are found in a normal mode can be translated into the original modes. Such a quantum noise description of the effect allows us to appreciate the localization of the relatively strong particle correlations about the polariton branches.

Due to strong dissipation channels and relatively weak coupling strengths, the steady state exhibits a low level of quantum correlations among the subsystems. On the other hand, some of its promising features can be found in early time quantum dynamics of the system. Our objective in the next section would be to go beyond steady state and explore some nonequilibrium cases for our model.

\section{Nonequilibrium coherence and correlations}

We investigate dynamics of quantum correlations among the radiation modes in the single ensemble case for the initial state $|\psi(0)\rangle=|11 g\rangle$. Truncating the Fock space for each radiation mode at seven photons, the quantum master equation is solved by the direct integration.

To comprehend clearly the effect of $g$ on the correlation dynamics, we first consider the case $\kappa_{a}, \kappa_{b}, \gamma, \gamma_{\phi}=0$ and, accordingly, $E_{a}, b=0$. We choose $\delta_{b}=0$ for which the probe resonance with the upper polariton branch is at $\delta=\sqrt{J^{2}+g^{2}}$.

By calculating the $\xi_{x}^{2}$, we find that there is no multiparticle entanglement for $g \leqslant J$. We examine $\xi_{x}^{2}$ for $g>J$ in Fig. 5 . We plot $-\log _{2} \xi_{x}^{2}$ so any positive value of it would be the indicator of the particle entanglement. The strongest multiparticle correlations exist when $g \sim J$. Further increase of $g$ gives weaker squeezing. While the entanglement happens earlier at larger $g$, its duration severely decreases with $g$.

In the regime $g<J$ lacking multiparticle entanglement, there is strong mode entanglement as depicted in the first column of Fig. 6, where we plot the $S$ together with the fidelities $F_{j}=\operatorname{Tr}\left(\left|\psi_{j}\right\rangle\left\langle\psi_{j}\right| \rho\right)$ of the states $\left|\psi_{j=0, \ldots, 4}\right\rangle=$ $|11 g\rangle,|10 e\rangle,|20 g\rangle,|02 g\rangle,|01 e\rangle$, respectively. For $g \sim 0$ there are two maximally entangled two-particle states [10]. The one appearing at the peaks $S \sim 1.5$ is $|\psi\rangle=(|20 g\rangle+|02 g\rangle \pm$ $i|11 g\rangle) / \sqrt{3}$. It is entangled in three-dimensional space so the maximum $S=\log _{2}(3) \sim 1.5$, whereas the one at the peaks $S \sim 1$ is of the form $|\psi\rangle=(|20 g\rangle+|02 g\rangle) / \sqrt{2}$ and is entangled in two-dimensional state space. At regular times

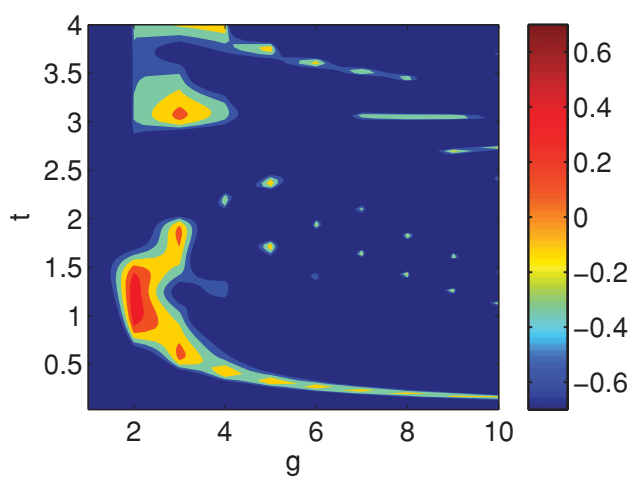

FIG. 5. (Color online) Time and $g$ dependence of $\xi_{x}^{2}$ when there is no dissipation in the system for an initial state $|\psi(0)\rangle=|11\rangle_{a b}|g\rangle$ at $\delta_{b}=0$ and $\delta=\sqrt{g^{2}+J^{2}}$ with $J / 2 \pi=1 \mathrm{MHz}$. In the figure $g / 2 \pi$ is in $\mathrm{MHz}$ and $t$ is in microseconds.
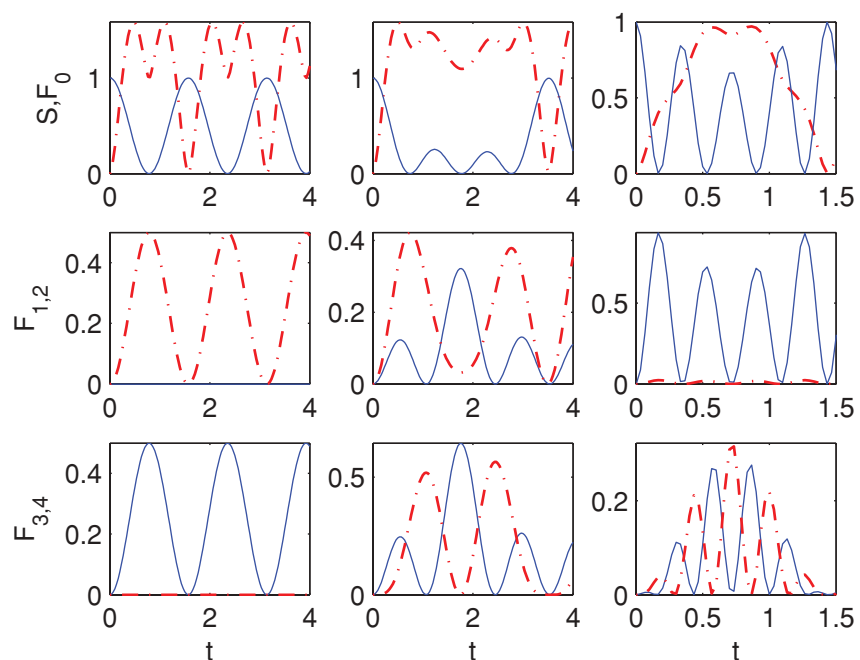

FIG. 6. (Color online) Time dependence of $S$ and fidelities $F_{j}, j=0,1,2,3,4$ of the states $|11 g\rangle,|10 e\rangle,|20 g\rangle,|02 g\rangle,|01 e\rangle$, respectively. There is no dissipation in the system and the initial state is $|\psi(0)\rangle=|11\rangle_{a b}|g\rangle . \delta_{b}=0$ and $\delta=\sqrt{g^{2}+J^{2}}$ with $J / 2 \pi=1 \mathrm{MHz}$ are used. In the figure $t$ is in microseconds. The left, middle, and right columns are for $g / 2 \pi=0,1,10 \mathrm{MHz}$. Dash-dotted curves in the first, second, and third rows are for $S, F_{2}$, and $F_{4}$

the system is disentangled to $\left|\psi_{0}\right\rangle$. Strong and long-lived mode entanglement can be found when $g \sim J$ as shown in second column of Fig. 6. Splitting of the degenerate states helps to preserve the three-dimensional structure of the subspace of entanglement to ensure the highest available $S \sim 1.5$. In addition, destruction of the disentanglement ensures that high $S$ can be maintained relatively longer in comparison to the $g \sim 0$ regime. For $g \gg J$, according to the last column of Fig. 6, the dynamical behavior of $S$ resembles qualitatively the $g \sim 0$ regime but for the lower $S \sim 1$ values. The states at these points are constructed by $|11 g\rangle$ and $|01 e\rangle$ for the middle and lower peaks and by $|10 e\rangle$ and $|02 g\rangle$ for the higher peaks. They are both in two-dimensional-state spaces so that $S \sim 1$.

We now examine the behavior of correlations in the presence of dissipation. A typical result for our simulations is shown in Fig. 7, indicating the dynamical behavior of

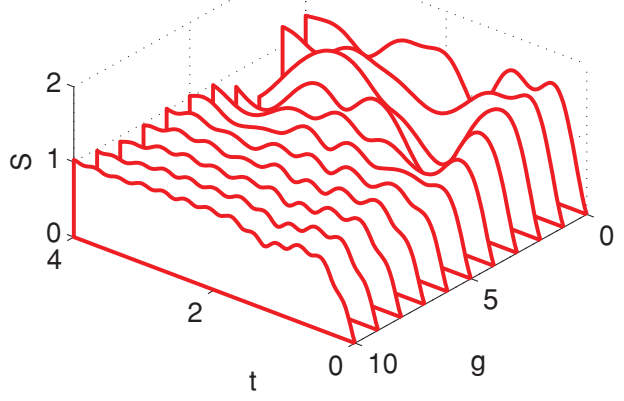

FIG. 7. (Color online) Time and $g$ dependence of von Neumann entropy for an initial state $|\psi(0)\rangle=|11\rangle_{a b}|g\rangle$ at $\delta=\sqrt{g^{2}+J^{2}}$ and $\delta_{b}=0$. In the figure $g / 2 \pi$ is in megahertz and $t$ is in microseconds. The decay rates are $\kappa_{a} / 2 \pi, \kappa_{b} / 2 \pi, \gamma_{\phi} / 2 \pi=0.1 \mathrm{MHz}$. 


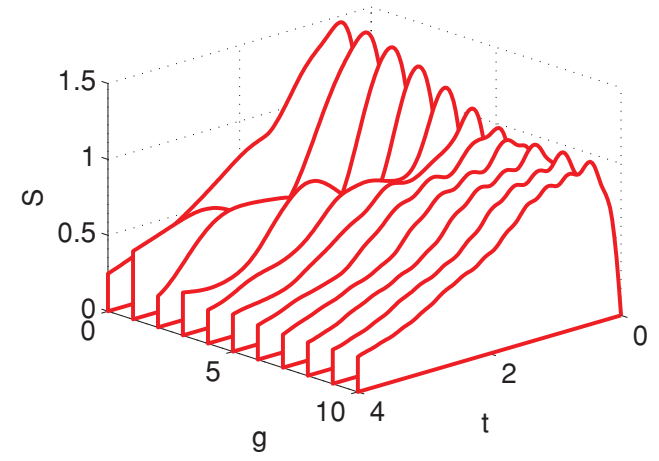

FIG. 8. (Color online) Same as shown in Fig. 7 but for $\kappa_{a} / 2 \pi=$ $0.4, \kappa_{b} / 2 \pi=0.4, \gamma_{\phi} / 2 \pi=0.3 \mathrm{MHz}$ and $\delta_{b}=J$.

$S$ for the same parameters with the lossless case but for $\kappa_{a} / 2 \pi, \kappa_{b} / 2 \pi, \gamma_{\phi} / 2 \pi=0.1 \mathrm{MHz}$. If we use the larger decay rates, the same as those used in the steady-state analysis, and take the same $\delta_{b}=J$ with the steady-state case, we find the result shown in Fig. 8. We see that the features of mode entanglement discussed in dissipationless case is vastly available in the case of moderate damping. In the larger damping case, except the early times, entanglement cannot be obtained. Some signatures of the prolonged entanglement duration are still found in the $g \sim J$ even under such strong damping conditions.

Figures 9 and 10 show that multiparticle entanglement can still be found at certain $g$ values at particular time domains for weaker damping situations, whereas no spin squeezing and entanglement remain in the larger dissipation case.

\section{B. Two atomic ensembles}

We shall now consider the case of two identical, symmetrically placed cyclic ensembles. In contrast to $a-b$ coupling, there is no direct $a_{1}-a_{2}$ interaction now. We did not find multi-partite entanglement between the photons of the $a_{1}$ and $a_{2}$ modes. We focus on mode entanglement and population dynamics in this case. Since the system has three mode radiation modes, we take into account the possibility of genuine three-mode entanglement. We use an extended set

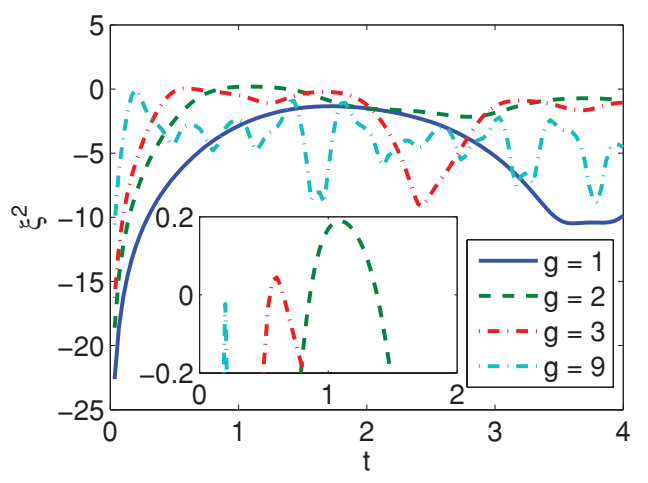

FIG. 9. (Color online) Same as shown in Fig. 7 but for the squeezing parameter $\xi_{x}^{2}$.

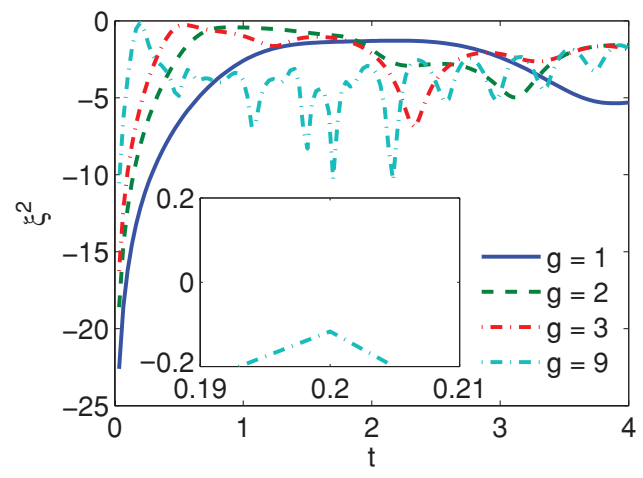

FIG. 10. (Color online) Same as shown in Fig. 8 but for the squeezing parameter $\xi_{x}^{2}$.

of mode correlation parameters [43]

$$
\begin{aligned}
& \lambda_{1 b}=\left|\left\langle a_{1}^{\dagger} b\right\rangle\right|^{2}-\left\langle a_{1}^{\dagger} a_{1} b^{\dagger} b\right\rangle, \\
& \lambda_{2 b}=\left|\left\langle a_{2}^{\dagger} b\right\rangle\right|^{2}-\left\langle a_{2}^{\dagger} a_{2} b^{\dagger} b\right\rangle, \\
& \lambda_{12}=\left|\left\langle a_{1}^{\dagger} a_{2}\right\rangle\right|^{2}-\left\langle a_{1}^{\dagger} a_{1} a_{2}^{\dagger} a_{2}\right\rangle .
\end{aligned}
$$

When any two of them become simultaneously positive, genuine three-mode entanglement is found. Typical example is the so-called three-parity $W$ state [29], which is defined to be $W=(|100\rangle+|010\rangle+|001\rangle) / \sqrt{3}$. In our case the Hilbert space is enlarged by the two-level atom. We denote basis states with $\left|n_{1}, n_{b}, n_{2}, s\right\rangle$, where $n_{1,2}, n_{b}$ are the occupation numbers of the radiation modes $a_{1,2}, b$ and $s=e, g$.

We solve the density matrix equations using quantum trajectory method. We take 25 trajectories and truncate Fock space at two photons in each radiation mode $a_{1,2}, b$. We fix $\delta_{b}=0$ and $\delta=\sqrt{J^{2}+g^{2}}$ for a given $g$ for which the probes are in resonance with the upper polariton branch of the single excitation manifold. For arbitrary $\Delta$ there are four such branches now due to additional optical degrees of freedom in comparison to the single-ensemble case with three polariton branches. We explore dynamical evolution of two initial preparations of the system: (i) $|\psi(0)\rangle=|010 g\rangle$ and (ii) $|\psi(0)\rangle=|100 g\rangle$.

In the first case, our simulations reveal that when $g<J$, the population of the microwave cavity photon is symmetrically split into to the optical modes, as shown in Fig. 11. The effect is an optical analog of coherent population trapping [49]. At large $g>J$, splitting is suppressed and the cavity photon is makes predominantly localized interactions with the two-level atom. To comprehend this effect let us write the dark state $|\mathrm{DS}\rangle$ in the single excitation manifold for the two-ensemble model as follows:

$$
|\mathrm{DS}\rangle=\frac{1}{\sqrt{J^{2}+g^{2}}}\left(-J \sigma^{\dagger}-\frac{g}{\sqrt{2}} a_{1}^{\dagger}-\frac{g}{\sqrt{2}} a_{2}^{\dagger}\right)|\mathrm{vac}\rangle,
$$

with $|\mathrm{vac}\rangle=|000 \mathrm{~g}\rangle$ being the vacuum state of the composite system. For $g \gg J$, $|\mathrm{DS}\rangle$ becomes $|\mathrm{DS}\rangle \approx-(|100 g\rangle+$ $|001 g\rangle) / \sqrt{2}$, whereas for $g \ll J$ it gets $|\mathrm{DS}\rangle \approx|000 e\rangle$. This suggests that in the regimes where the long-range interaction $J$ dominates over the short-range interaction $g$, the dark state $|000 e\rangle$ is inaccessible by the initial state $|\psi(0)\rangle=|010 \mathrm{~g}\rangle$. As such, $|\psi(0)\rangle$ yields only a coherent transfer of $b$ population 
(a)
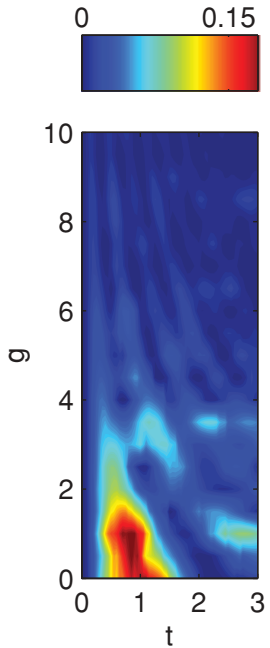

(b)
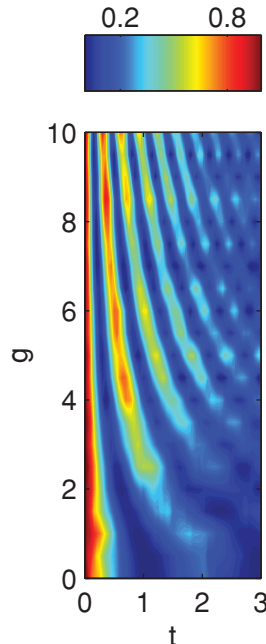

(c)
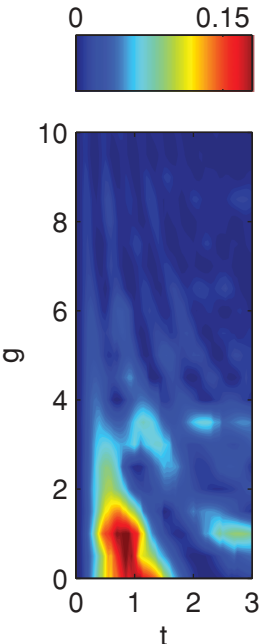

FIG. 11. (Color online) Time dependence of populations of the radiation modes (a) $n_{1}=\left\langle a_{1}^{\dagger} a_{1}\right\rangle$, (b) $n_{b}=\left\langle b^{\dagger} b\right\rangle$, and (c) $n_{2}=\left\langle a_{2}^{\dagger} a_{2}\right\rangle$ for an initial state $|\psi(0)=| 010 g\rangle$. We take $J_{1,2} / 2 \pi=1 \mathrm{MHz}$, $\delta_{b}=0, \delta=\sqrt{g^{2}+J^{2}}, \kappa_{1,2, b}=(2 \pi) 0.4 \mathrm{MHz}, \gamma / 2 \pi=0.02 \mathrm{MHz}$, $\gamma_{\phi} / 2 \pi=0.3 \mathrm{MHz}, E_{x}=0.1 \kappa_{x}, x=1,2, b$.

to the $a_{1,2}$ modes. According to Fig. 11 population transfer is complete in about $t \sim 1 \mu \mathrm{s}$. Population exchange is a coherent process happening at regular time intervals of every $2 \mathrm{~J}$. At lower damping rates, such as $\gamma, \kappa_{1,2, b} / 2 \pi \sim 0.1 \mathrm{MHz}$, we find a few more cycles of transfer.

When the tunneling from $b$ to $a_{1,2}$ is complete, the system radiates genuine two-mode entangled optical photons which can be verified by Fig. 12. We verified that $S \sim 1$ is a reliable bipartite entanglement signature at the corresponding times for low $\left(\gamma, \kappa_{1,2, b} / 2 \pi \sim 0.1 \mathrm{MHz}\right)$ damping. During the course of transfer, at about $t \sim 0.5 \mu \mathrm{s}$ all three modes are populated. At such times, as the excited state of the two-level atom remains in the dark, a three-parity $W$ state with genuine three-mode entanglement is found. The corresponding

(a)


(b)
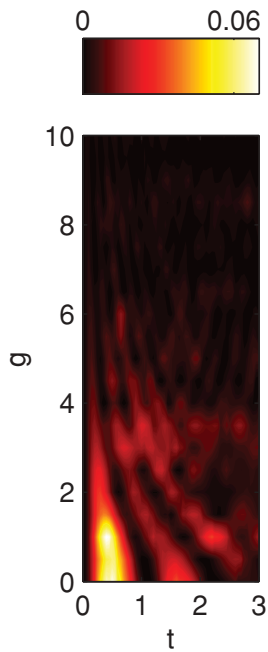

(c)
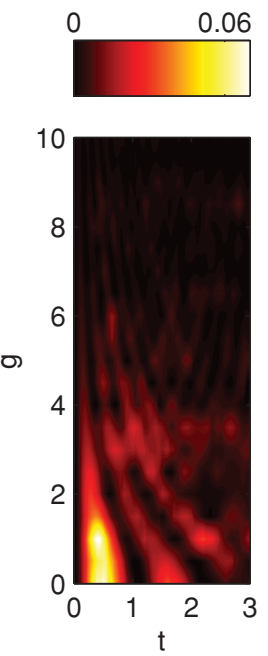

FIG. 12. (Color online) Same as shown Fig. 11 but for the genuine mode entanglement parameters (a) $\lambda_{12}$, (b) $\lambda_{2 b}$, and (c) $\lambda_{1 b}$.

(a)


(b)
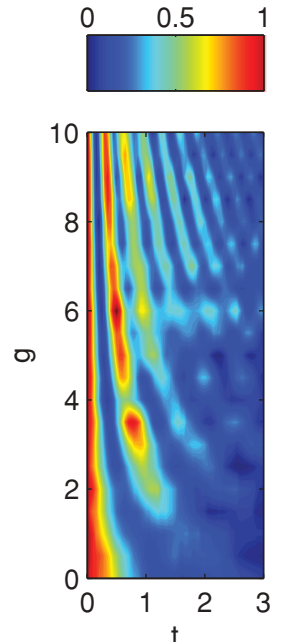

(c)
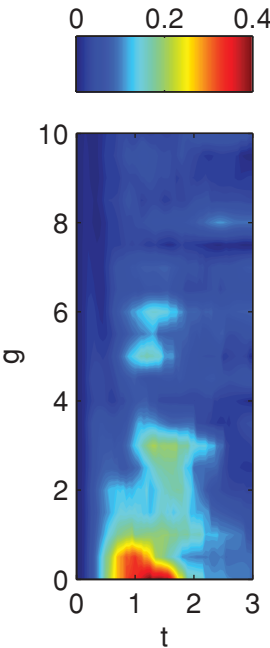

FIG. 13. (Color online) Same as shown in Fig. 11 but for an initial state $|\psi(0)=| 110\rangle|g\rangle$.

$S \sim 0.7$ is relatively lower than the maximally entangled two-mode state. Due to its robustness against single-particle losses relative to Greenberger-Horne-Zeilinger (GHZ) states $[50,51](|\mathrm{GHZ}\rangle=(|000\rangle+|111\rangle) / \sqrt{2})$ and its potential role in quantum networks, $W$ states have appealing features in quantum information science. They are proposed for bipartite entanglement distillation [52]. Our results provide a promising setting to realize these goals.

In the $g \gg J$ case, $|\mathrm{DS}\rangle \approx-(|100 \mathrm{~g}\rangle+|001 \mathrm{~g}\rangle) / \sqrt{2}$ remains in the dark. The initial state is predominantly coupled to $|000 e\rangle$. The radiation energy is trapped in the microwave cavity $(b)$ mode and exchanged locally with the two-level atom. Associated Rabi oscillations are shown in the middle figure of Fig. 11. Mode entanglement parameters practically vanish in this case, as shown in Fig. 12.

We consider an asymmetric populated initial state to show that instead of two-mode entanglement of optical modes, optical- and cavity-mode entanglement can be emphasized. For this aim we start with $|\psi(0)\rangle=|110 g\rangle$. Population dynamics at various $g$ are depicted in Fig. 13.

This localization of the cavity photon in the $g>J$ Rabi oscillations regime can be used to block an optical mode to access the other distant optical mode and, as such, the corresponding distant ensemble. The localizations of the radiation energies in $g \gg J$ regime can be seen as blocking of an optical mode interacting with an ensemble to access a distant ensemble and the corresponding optical mode via the microwave nonlinear cavity. In the $g \ll J$ limit, the $b$ mode still splits into two but its tunneling into $a_{1}$ is balanced with the tunneling of $a_{1}$ into $b$. There is no net population current between the $a_{1}$ and $b$ modes so we see no change of population of $a_{1}$ mode in Fig. 13(c), except its usual decay. Accordingly, $\lambda_{12}<0$ and $\lambda_{1 b}<0$, as indicated by the white regions about $t \sim 0.5 \mu \mathrm{s}$ in Figs. 14(a) and 14(c), whereas Fig. 14(b) demonstrates that there is genuine two-mode entanglement between the $a_{2}$ and $b$ modes at this time. In contrast to the previous $W$ state arising at this time with three-mode entanglement, the initial imbalance of the populations in the present case effectively removes one 
(a)


(b)

0

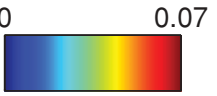

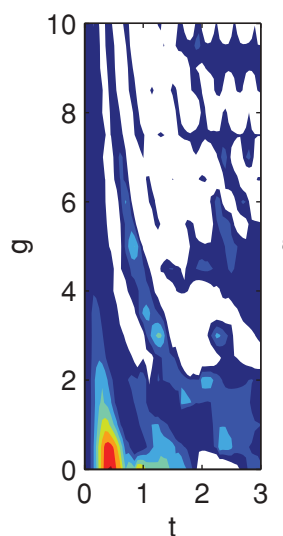

(c)
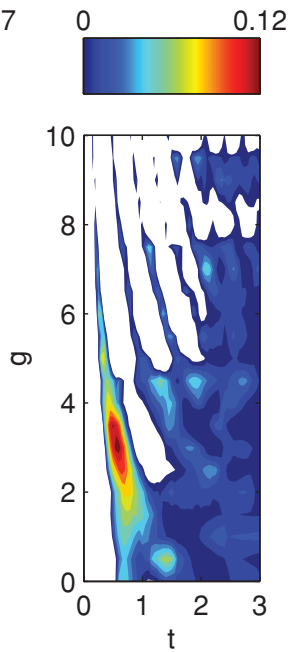

FIG. 14. (Color online) Three-mode correlation parameters that can be used to reveal genuine three-mode entanglement in the evolution of an initial state $|\psi(0)=| 110\rangle|g\rangle$.

particle from the $W$ state to distill or concentrate a two-mode entanglement from it. At $t=1 \mu \mathrm{s}$ the situation is similar to the previous symmetric initial state and we see two-mode entanglement between the optical $a_{1}$ and $a_{2}$ modes. The effect can be viewed as the presence of dynamically distinct and transferable bipartite entanglement phases in the multimode system. While we consider here only the simplest symmetric and identical two-ensemble, the time-dependent, controllable different interaction coefficients $J_{1}, J_{2}$, and $g$ allow for more rich coherent information and population transfer protocols.

\section{PROPOSAL OF EXPERIMENTAL IMPLEMENTATION}

In order to realize the model Hamiltonians discussed in the preceding sections, now we shall consider a straightforward extension of a recent experiment demonstrating strong coupling of an $\mathrm{N}-\mathrm{V}$ center ensemble to a coplanar waveguide resonator [5]. The setting is shown in Fig. 15, where two diamond crystals and a transmon qubit [53-55] are strongly coupled to microwave stripline cavity [23]. Diamond crystals containing color center defects serve as the quantum memory, and the transmon qubit serves as the quantum hardware for rapid quantum information processing. The microwave cavity photons serve as the local data bus. By interfacing this device with external optical fields, such a stationary quantum information unit would have access to optical flying qubits

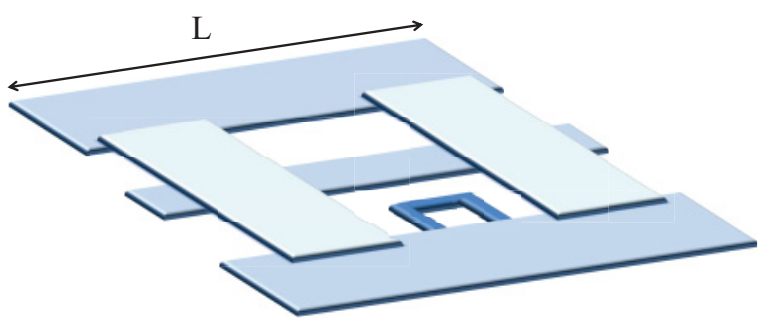

FIG. 15. (Color online) Transmon qubit and two diamond crystals couple to microwave stripline cavity of lenght $L$ and width $d$. for quantum networking and communication at free space communication wavelengths.

Strong coupling of a single diamond with N-V centers to a superconducting CPWG resonator has been shown recently [5]. We propose a a straightforward extension of the physical system of Ref. [5] to the case of two diamond crystals. In order to place the diamond crystals to locations where the magnetic field is strong and homogeneous, and the transmon qubit to a similar location but for the electric field, a $L=\lambda$ cavity is considered suitable for our purpose. The microwave wavelength for the fundamental frequency of the cavity $\omega_{1} / 2 \pi \sim 6 \mathrm{GHz}$ is $\lambda \sim 50 \mathrm{~mm}$. The crystals are assumed at the same size of $3 \times 3 \times 0.5 \mathrm{~mm}^{3}$. The canonically quantized magnetic field of the fundamental quasi-TEM mode of the cavity is given in the laboratory (cavity) frame by

$$
\vec{B}(x, y, z)=\vec{B}_{\perp}(x, y)\left(b+b^{\dagger}\right) \cos \frac{\pi z}{L},
$$

where $\vec{B}_{\perp}(x, y)$ is the transverse field distribution. Placement of the crystals $\lambda / 2$ apart, at the magnetic field antinodes of the fundamental mode along the cavity axis $(x)$, allows for sufficiently strong magnetic coupling to the cavity mode as well as adequate space for optical access to each crystal. At the same time, a transmon qubit placed in the middle of the crystals would be at the electric field antinode of the fundamental cavity mode. It is now well established that, under certain conditions on the Cooper-pair box and circuit QED system, the Jaynes-Cummings model can describe the coupling of the qubit to the single mode cavity $[53,56]$.

In order to determine the conditions to obtain the model Hamiltonians we have used in the preceding sections, we shall focus here on the relatively less explored N-V center coupling to the cavity; in particular, we examine if such a coupling can be described by the $\Delta$-type transition model.

The diagram shown in Fig. 16 describes the relevant energy levels of a negatively charged N-V center $[57,58]$. The groundstate manifold is ${ }^{3} A$, with zero-field splitting $D \sim 2.87 \mathrm{GHz}$; the excited state is ${ }^{3} E$ separated from the ground level by the zero phonon line at $637 \mathrm{~nm}$; and there is a metastable state ${ }^{1} A$ with nonradiative transitions. Further splittings of the ${ }^{3} E$ level are on the order of 2.6 and $2.3 \mathrm{GHz}$ for ${ }^{3} E_{x}$ and ${ }^{3} E_{y}$, respectively.

Electric dipole $(E 1)$ and magnetic dipole $(M 1)$ selection rules forbid an immediate realization of Raman-type $(\Lambda)$ transitions in the given energy-level diagram, let alone a $\Delta$ transition. On the other hand, optical Raman-induced

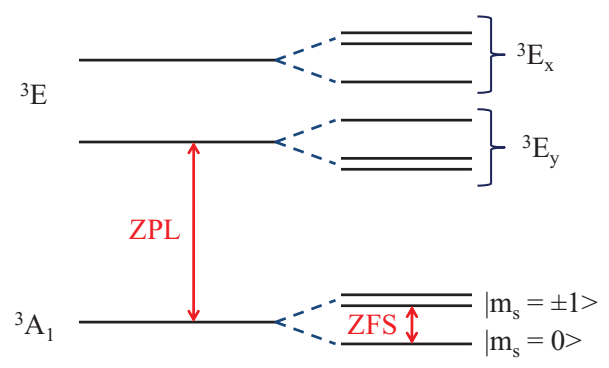

FIG. 16. (Color online) Energy-level diagram of negatively charged N-V center. ZPL is the zero phonon line at $638 \mathrm{~nm}(1.945 \mathrm{eV})$ and ZFS is the zero-field splitting at $2.87 \mathrm{GHz}$. 




FIG. 17. (Color online) Energy-level diagram of a negatively charged $\mathrm{N}-\mathrm{V}$ center. ZPL is the zero phonon line at $638 \mathrm{~nm}(1.945 \mathrm{eV})$ and ZFS is the zero-field splitting at $2.87 \mathrm{GHz}$.

spin-flipping processes [59-61] have been realized in addition to spin-conserving (cyclic) transitions [62] in recent experiments. To comprehend how this happens, detailed recent studies of the excited level reveal that among the potential spinspin and spin-orbit interactions that can influence the excited level structure, it is the local strain-induced level mixing that allows for the essential non-spin-preserving transitions for an optical $\Lambda$ scheme. Using external magnetic or electric fields or specifically engineered $\mathrm{N}-\mathrm{V}$ centers, one can thus produce the level combinations for such transitions. To make our discussion concrete, we generalize the $\Lambda$-transition generation protocol using external electric fields [63] to the case of the $\Delta$ transition. Typical electric field strengths of several megavolts per meter are experimentally used [61]. Under a strong electric field the level structure of the negatively charged $\mathrm{N}-\mathrm{V}$ center is shown in Fig. 17. Here, both cyclic (spin-preserving) and spin-flipping transitions can simultaneously happen.

Optical fields are right circular polarized. E1 and ESR selection rules allow us to obtain cyclic transitions in this single $\mathrm{N}-\mathrm{V}$ molecule in a hexagon scheme, where all nodes can be accessed starting from any other node, as shown in Fig. 17(b). The upper level $A$ is an excited level generated by the mixed states by the electric field. The intermediate levels are $\left|m_{s}=-1, m_{I}=\uparrow\right\rangle$ and $\left|m_{s}=+1, m_{I}=\downarrow\right\rangle$, denoted by $|-\rangle$ and $|+\rangle$, respectively. The lowest levels are $\left|m_{s}=0, m_{I}=\uparrow\right\rangle$ and $\left|m_{s}=0, m_{I}=\downarrow\right\rangle$, denoted by $|\uparrow\rangle$ and $|\downarrow\rangle$, respectively. Coupling of the cavity field to the ground state ${ }^{3} \mathrm{~A}$ manifold is described by an anomalous Zeeman interaction of the form

$$
H=\sum_{i} \sigma_{i}^{T} \tilde{D} \sigma_{i}+\mu_{B} \sum_{i} \vec{B}_{i}^{T} \tilde{g} \vec{\sigma}_{i}
$$

where $\mu_{B}$ is the electronic Bohr magneton and $\tilde{g}$ is a diagonal tensor with elements $\left(g_{\perp}, g_{\perp}, g_{\|}\right)$with $g_{\perp}=2.0024$ and $g_{\|}=2.0028$ in a frame where the quantization axis $(z)$ is chosen to be the symmetry axes of the N-V molecule. In the $C_{3 v}$ symmetry of diamond, one can find four of such axes corresponding to four crystallographic classes $N V-f, f=$ $1,2,3,4$. We work within a particular class, but omit its label $(f)$ for the sake of notational simplicity. Expressions for different classes can be obtained by using spherical tensor operations. The nuclear Zeeman term is too weak and thus is ignored. Depending on the relative density of surrounding nuclei $\left({ }^{1} 4 \mathrm{~N},{ }^{1} 5 \mathrm{~N},{ }^{1} 3 \mathrm{C}\right)$, quadrupole and hyperfine interactions can impose significant decoherence on the central electronic spin [5]. Their contribution can be attributed to our effective loss terms in the master equation treatment we used. The first term is the zero-field Hamiltonian, with $\tilde{D}$ being the zero-field splitting tensor, which is diagonal in the molecular frame with the elements $\left(D_{\perp}, D_{\perp}, D_{\|}\right)$. It simplifies to $H_{\mathrm{ZFS}}=D \sigma_{z}^{2}$, up to a constant, with $D=3 D_{\|} / 2 \sim 2.87 \mathrm{GHz}$. The index $i=$ $1, \ldots, N_{f}$ is the index of N-V defects at location $\vec{r}_{i}=\left(x_{i}, y_{i}, z_{i}\right)$ in the laboratory (cavity) frame. We shall consider a single crystal case first.

Writing the cavity field as $\vec{B}\left(\vec{r}_{i}\right)=\overrightarrow{\mathcal{B}}_{i}\left(b+b^{\dagger}\right)$, with $\overrightarrow{\mathcal{B}}_{i}=\vec{B}_{\perp}\left(x_{i}, y_{i}\right) \cos \left(\pi z_{i} / \lambda\right)$, the Hamiltonian becomes in the ground-state basis

$$
H=D \sum_{i= \pm} X_{i i}+g_{B}\left(b+b^{\dagger}\right)\left(X_{\downarrow+}+X_{\uparrow-}+\text { H.c. }\right),
$$

where we neglected the small Zeeman shift, which is on the order of kilohertz for a cavity field in the order of nT, next to the $D \sim \mathrm{GHz}$. The generalized Hubbard operators are introduced to be

$$
\begin{gathered}
X_{\downarrow+}=\sum_{i} \frac{\mathcal{B}_{i+}}{\mathcal{B}_{\perp}} R_{\downarrow+}^{(i)}, \\
X_{\uparrow-}=\sum_{i} \frac{\mathcal{B}_{i-}}{\mathcal{B}_{\perp}} R_{\uparrow+}^{(i)}, \\
X_{+-}=\sum_{i} R_{+-}^{(i)} .
\end{gathered}
$$

Here, $\mathcal{B}_{i \pm}=\mathcal{B}_{i x} \pm \mathcal{B}_{i x}$ and

$$
\mathcal{B}_{\perp}=\sqrt{\sum_{i}\left(\mathcal{B}_{i x}^{2}+\mathcal{B}_{i y}^{2}\right)} .
$$

In the large $N_{f}$ and weak excitation limit, for a quasiuniform magnetic field distribution, we can employ the group contraction method as before to get a bosonic representation for the Hubbard operators such that $X_{\downarrow+} \sim B_{+}$and $X_{\uparrow-} \sim B_{-}$. Consistently, they form the Schwinger representation of the remaining isospin subgroup so we take $X_{+-} \sim B_{+}^{\dagger} B_{-}$which allows for replacing $X_{++}+X_{--}$with $B_{+}^{\dagger} B_{+}+B_{-}^{\dagger} B_{-}$in the zero-field splitting term of the Hamiltonian.

The interaction of the excited state with the optical fields can be described in terms of the collective operators as well. To make this consistently with the microwave transitions we first make the usual bosonization of ground level to excited level $|A\rangle$ transition via $X_{\uparrow A} \sim X_{\downarrow A} \sim A$. Here we implicitly assume the populations in degenerate ground levels are large and the $A$ mode is weakly excited. Then consistency with the isospin groups is satisfied by taking their Schwinger representations as $X_{A \pm}=A^{\dagger} B_{ \pm}$. To achieve this limit adequately, we demand that the optical fields are to be made sufficiently homogeneous and isotropic over the diamond substances. This may be accomplished by suitable confocal microscopy. The total Hamiltonian in this bosonic limit describes again a cyclic transition system which is reduced from a hexagon to a 
diamond scheme, as shown in Fig. 17(c). The degeneracy of intermediate levels allows us to employ a simple multilevel Morris-Shore transformation [64-66] in the form $B=\left(B_{-}+\right.$ $\left.B_{+}\right) / \sqrt{2}$ and $B^{\prime}=\left(B_{-}-B_{+}\right) / \sqrt{2}$. Geometrically, this folds the diamond scheme into a delta-type cyclic transition as shown in Fig. 17(d), where $B^{\prime}$ is uncoupled from the system.

Even though we have described how to get a cyclic $\mathrm{N}-\mathrm{V}$ ensemble, our analysis so far is limited to a single crystallographic class. To complete our discussion let us now address the case of all $\mathrm{N}-\mathrm{V}$ classes. For that aim we make use of slightly more complicated Morris-Shore transformations.

The Hamiltonian, including all the N-V classes, is

$$
\begin{aligned}
H= & \varpi_{A} \sum_{f=1}^{4} A_{f}^{\dagger} A_{f}+\omega_{B} \sum_{f=1}^{4} B_{f}^{\dagger} B+\omega_{a} a^{\dagger} a+\omega_{b} b^{\dagger} b \\
& +\frac{\omega_{0}}{2} \sigma_{z}+g\left(b^{\dagger} \sigma+\text { H.c. }\right)+\Omega \sum_{f=1}^{4}\left(A_{f}^{\dagger} B_{f}+\text { H.c. }\right) \\
& +\sum_{f=1}^{4}\left(g_{B f} b B_{f}^{\dagger}+g_{A f} a A_{f}^{\dagger}+\text { H.c. }\right) .
\end{aligned}
$$

We find that under the conditions

$$
\frac{g_{B 2}}{g_{B 1}}=\frac{g_{A 2}}{g_{A 1}}, \quad \frac{g_{B 3}}{g_{B 1}}=\frac{g_{A 3}}{g_{A 1}}, \quad \frac{g_{B 4}}{g_{B 1}}=\frac{g_{A 4}}{g_{A 1}},
$$

only two quasi-spin-wave modes can be coupled to the electromagnetic modes. These so-called "bright" modes are given by collective bosonic modes

$$
B=\sum_{f=1}^{4} \frac{g_{B f}}{g_{B}} B_{f}, \quad A=\sum_{f=1}^{4} \frac{g_{A f}}{g_{A}} A_{f},
$$

where

$$
g_{A}=\sqrt{\sum_{f=1}^{4} g_{A f}^{2}}, \quad g_{B}=\sqrt{\sum_{f=1}^{4} g_{B f}^{2}} .
$$

The uncoupled, "dark" modes are listed in Appendix B. After that we arrive at our starting Hamiltonians, Eqs. (4) and (5) in Sec. II. A simple way to realize the dark quasi-spin-wave modes is to make the interaction coefficients $g_{A f}$ and $g_{B f}$ equal for each $\mathrm{N}-\mathrm{V}$ class. This is the case in the experiment of strong coupling of the N-V center and superconducting cavity. By choosing a particular placement of the diamond crystal over the cavity substrate for which the cavity magnetic field makes effectively equal angles for the four quantization axes of the $\mathrm{N}-\mathrm{V}$ classes, the Zeeman interaction coefficients become equal [5]. The collective enhancement of the interactions would depend on $N_{f}$, which we may assume $N_{f}=N / 4$. Finally, the case of two diamond crystals can be immediately generalized from these results, where only the magnetic field would change a sign at the location of the other crystal half wavelength away.

The typical parameters in these models are, according to recent experiments as discussed in Ref. [67], as follows. Qubit decoherence times are on the order of a few microseconds, in particular, that for the transmon is about $\sim 4 \mu \mathrm{s}$ [53]. For $T_{1} \sim 7 \mu \mathrm{s}$ and $T_{2} \sim 500 \mathrm{~ns}$, relaxation and dephasing rates are respectively $\gamma_{1} / 2 \pi \sim 0.02 \mathrm{MHz}$ and $\gamma_{\phi} / 2 \pi \sim 0.31$ $\mathrm{MHz}$ and the resonance frequency of the qubit is tunable in the ranges $\omega_{0} \sim 5-15 \mathrm{GHz}$. The stripline cavity loss rate is $\kappa / 2 \pi=\omega_{b} / 2 \pi Q \sim 5 \mathrm{kHz}$ at $\omega_{b} / 2 \pi=5 \mathrm{GHz}$ in a cavity with $Q$ factor $Q=10^{6}$. The cavity frequency is adjustable in the range of usually about $\omega_{b} / 2 \pi=5-10 \mathrm{GHz}$. Coupling strength between the cavity and the qubit can be adjusted in the ranges $g \sim 5-200 \mathrm{MHz}$. In our numerical simulations we choose our parameter ranges to be compatible within these practical values. In particular, we fix $g_{a} / \Omega=0.1$ so $J=-0.1 g_{b}, E_{a}=0.1 \Omega_{A}$. For $g_{b}=(2 \pi) 11 \mathrm{MHz}$ of Ref. [5], we take $J / 2 \pi=1 \mathrm{MHz}$. We assume $\Omega_{A}$ and the detunings are adjusted to make $E_{a}=E_{b} . E_{b}=\sqrt{N_{\mathrm{cav}}} \kappa_{b}$ with $N_{\mathrm{cav}}=0.01$ is cavity photon number for the empty microwave cavity on probe resonance under weak drive conditions. We use $\gamma \equiv \gamma_{1} / 2 \pi=0.02 \mathrm{MHz}$ and $\gamma / 2 \pi=0.3 \mathrm{MHz}$. We consider ranges of $g / 2 \pi$ up to $10 \mathrm{MHz}$ and $\delta / 2 \pi$ within $(-5,5)$ $\mathrm{MHz}$. To take into account the conditions of experiment Ref. [5] we use $\kappa_{b} / 2 \pi=0.4 \mathrm{MHz}$, while the effective loss of optical modes is chosen to be the same $\kappa_{a}=\kappa_{b}$, which corresponds to $\gamma_{A} / 2 \pi \sim 40 \mathrm{MHz}$ for the effective decay rate of the excited $\mathrm{N}-\mathrm{V}$ center, which is reasonable with the experiments [68].

The quantum master equation we have used can be reduced from a more general Bloch-Redfield master equation [69,70] under low temperature conditions [71].

\section{CONCLUSION}

Summarizing, we have investigated quantum statistical properties of optical radiation from $\Delta$-type cyclic atomic ensembles placed in a microwave cavity and coupled to a two-level atom. In particular, quantum coherence and quantum correlations of the emitted optical photons are examined. Effective models describing the system analogous to coupled driven, dissipative linear and nonlinear resonators are obtained under Fröchlich transformations.

It is shown that analogous to the adiabatic transfer protocol between stationary qubits [30], the quantum information can be transferred directly between the stationary qubit and the optical flying qubit. The proposed system completes the quantum memory and quantum hardware state transfer protocols by interfacing them with quantum communication channels. In addition, it is found that quantum coherence characteristics can be transferred between the microwave and optical fields.

Furthermore, it is shown that distant entanglement between two optical modes can be realized in the case of two-cyclic atomic ensembles. In addition, conditions of three-mode entanglement are also revealed. Controllable particle and mode entanglement for the optical modes or between the optical and the microwave photons is shown to be realizable on demand.

Finally, diamond crystals with N-V centers in superconducting coplanar waveguide resonators coupled to transmon qubits are proposed to physically realize the model system. The parameters used in numerical simulations are justified for the proposed system. Under a strong electric field, it is described that a single $\mathrm{N}-\mathrm{V}$ center allows for a particular hexagon-type cyclic transitions. Using a group contraction method for the ensemble case, the transition type reduces to 
a diamond type. For a general treatment of the N-V center, taking into account all four crystallographic classes and using generalized multilevel Morris-Shore transformations, a further reduction to a $\Delta$-type cyclic scheme is obtained.

We hope our work can inspire and contribute to ongoing efforts for interfacing optical networks with quantum memories and information processing units.

\section{ACKNOWLEDGMENTS}

Ö.E.M. gratefully acknowledges A. Atac Imamoğlu for suggesting this problem and for illuminating discussions. Useful discussions with E. Ilgunsatiroglu are acknowledged. This work is supported by D.P.T. (T.R. Prime Ministry State Planning Organization) under Project No. 2009K120200.

\section{APPENDIX A: COEFFICIENTS OF THE EFFECTIVE HAMILTONIAN}

Here we list the coefficients in Eq. (12) explicitly.

$$
\begin{aligned}
& \varpi_{a}^{\prime}==\varpi_{a}+g_{a}^{2} \sum_{\lambda} \frac{u_{\lambda}^{2}}{\varpi_{a}-\Omega_{\lambda}}, \\
& \omega_{b}^{\prime}=\omega_{b}+g_{b}^{2} \sum_{\lambda} \frac{v_{\lambda}^{2}}{\omega_{b}-\Omega_{\lambda}}, \\
& J=-\frac{g_{a} g_{b}}{2} \sum_{\lambda} u_{\lambda} v_{\lambda}\left(\frac{1}{\omega_{b}-\Omega_{\lambda}}+\frac{1}{\varpi_{a}-\Omega_{\lambda}}\right), \\
& \Omega_{\lambda}^{\prime}=\Omega_{\lambda}-g_{a} g_{b} \sum_{\lambda}\left(\frac{u_{\lambda} v_{\lambda}}{\omega_{b}-\Omega_{\lambda}}+\frac{u_{\lambda} v_{\lambda}}{\varpi_{a}-\Omega_{\lambda}}\right), \\
& Q=-\frac{1}{2} \sum_{\lambda \neq \mu}\left(\frac{g_{b}^{2} v_{\lambda} v_{\mu}}{\omega_{b}-\Omega_{\mu}}+\frac{g_{a}^{2} u_{\lambda} u_{\mu}}{\varpi_{a}-\Omega_{\mu}}\right), \\
& G_{\lambda}=-g g_{b} \frac{v_{\lambda}}{\omega_{b}-\Omega_{\lambda}}, \\
& E_{a}=\Omega_{A} g_{b} \sum_{\lambda} \frac{u_{\lambda}^{2}}{\varpi_{a}-\Omega_{\lambda}}, \\
& E_{b}^{\prime}=E_{b}+\Omega_{A} g_{b} \sum_{\lambda} \frac{u_{\lambda} v_{\lambda}}{\omega_{b}-\Omega_{\lambda}}, \\
& E_{p \lambda}=\Omega_{A} u_{\lambda}-E_{b} g_{b} \frac{v_{\lambda}}{\omega_{b}-\Omega_{\lambda}},
\end{aligned}
$$

where $\lambda, \mu= \pm$.
In the $\Omega \gg \Delta$ limit, these coefficients can be approximated to be

$$
\begin{aligned}
& \varpi_{a}^{\prime} \approx \varpi_{a}-\frac{1}{2} \frac{g_{a}^{2}}{\Omega^{2}}\left(\Delta_{a}-\frac{\Delta}{4}\right) \\
& \omega_{b}^{\prime} \approx \omega_{b}-\frac{1}{2} \frac{g_{b}^{2}}{\Omega^{2}}\left(\Delta_{b}+\frac{3 \Delta}{4}\right) \\
& J \approx \frac{g_{a} g_{b}}{\Omega}\left[1+\frac{\Delta^{2}}{16 \Omega^{2}}+\frac{\left(\Delta_{b}+\Delta / 2\right)^{2}+8\left(\Delta_{a}-\Delta / 2\right)^{2}}{2 \Omega^{2}}\right] \\
& \Omega_{\lambda}^{\prime} \approx \Omega_{\lambda}+\frac{g_{b}^{2}}{\Omega^{2}}\left(\Delta_{b}+\frac{3 \Delta}{4}\right)+\frac{g_{a}^{2}}{\Omega^{2}}\left(\Delta_{a}-\frac{\Delta}{4}\right) \\
& Q \approx-\frac{1}{2 \Omega^{2}}\left[g_{a}^{2} \Delta_{a}-g_{b}^{2} \Delta_{b}-\frac{\Delta}{2}\left(g_{a}^{2}+g_{b}^{2}\right)\right] \\
& G_{ \pm} \approx \pm \frac{g g_{b}}{\sqrt{2} \Omega}\left(1 \pm \frac{\Delta_{b}+3 \Delta / 4}{\Omega}\right) \\
& E_{a}=-\frac{\Omega_{A} g_{b}}{\Omega^{2}}\left(\Delta_{a}-\frac{\Delta}{4}\right), \\
& E_{b}^{\prime}=E_{b}-\frac{\Omega_{b} g_{b}}{\Omega}\left[1-\frac{\Delta^{2}+16\left(\Delta_{b}+\Delta / 2\right)^{2}}{16 \Omega^{2}}\right] \\
& E_{p \pm}=\frac{\Omega_{A}}{\sqrt{2}}\left(1 \mp \frac{\Delta}{4 \Omega}\right) \mp \frac{E_{b} g_{b}}{\sqrt{2} \Omega}\left(1 \pm \frac{\Delta_{b}+3 \Delta / 4}{\Omega}\right)
\end{aligned}
$$

\section{APPENDIX B: DARK QUASI-SPIN-WAVE MODES}

Here we list the dark quasi-spin-wave modes that are uncoupled from the $\mathrm{N}-\mathrm{V}$ ensemble Hamiltonian

$$
\begin{gathered}
B^{\prime}=\frac{g_{B 34}}{g_{B} g_{B 12}}\left(g_{B 1} B_{1}+g_{B 2} B_{2}\right)-\frac{g_{B 12}}{g_{B} g_{B 34}}\left(g_{B 3} B_{3}+g_{B 4} B_{4}\right) \\
B^{\prime \prime}=\frac{g_{B 2}}{g_{B 12}} B_{1}-\frac{g_{B 1}}{g_{B 12}} B_{2} \\
B^{\prime \prime \prime}=\frac{g_{B 4}}{g_{B 34}} B_{3}-\frac{g_{B 3}}{g_{B 34}} B_{4}
\end{gathered}
$$

where we define

$$
\begin{aligned}
& g_{B 12}=\sqrt{g_{B 1}^{2}+g_{B 2}^{2}}, \\
& g_{B 34}=\sqrt{g_{B 3}^{2}+g_{B 4}^{2}} .
\end{aligned}
$$

The same definitions and dark modes for the $A$-mode can be simply obtained by replacing $B \rightarrow A$ in these equations.

[3] A. Kuzmich et al., Nature 423, 731 (2003).

[4] M. Wallquist, K. Hammerer, P. Rabl, M. Lukin, and P. Zoller, Phys. Scr., T 137, 014001 (2009).

[5] Y. Kubo et al., Phys. Rev. Lett. 105, 140502 (2010).
[1] C. H. van der Wal et al., Science 301, 196 (2003).

[2] M. Nielsen and I. L. Chuang, Quantum Computation and Quantum Information (Cambridge University Press, Cambridge, UK, 2000). 
[6] D. I. Schuster et al., Phys. Rev. Lett. 105, 140501 (2010).

[7] H. Wu, R. E. George, J. H. Wesenberg, K. Molmer, D. I. Schuster, R. J. Schoelkopf, K. M. Itoh, A. Ardavan, J. J. L. Morton, and G. A. Briggs, Phys. Rev. Lett. 105, 140503 (2010).

[8] T. Duty et al., Physics 3, 80 (2010).

[9] Y. X. Liu, J. Q. You, L. F. Wei, C. P. Sun, and F. Nori, Phys. Rev. Lett. 95, 087001 (2005).

[10] Y. Li, L. Zheng, Y.-X. Liu, and C. P. Sun, Phys. Rev. A 73, 043805 (2006).

[11] A. Imamoğlu, Phys. Rev. Lett. 102, 083602 (2009).

[12] J. Siewert, T. Brandes, and G. Falci, Phys. Rev. B 79, 024504 (2009).

[13] G. Kurizki, M. Shapiro, and P. Brumer, Phys. Rev. B 39, 3435 (1989).

[14] E. Dupont, P. B. Corkum, H. C. Liu, M. Buchanan, and Z. R. Wasilewski, Phys. Rev. Lett. 74, 3596 (1995).

[15] R. Atanasov, A. Hache, J. L. P. Hughes, H. M. van Driel, and J. E. Sipe, Phys. Rev. Lett. 76, 1703 (1996).

[16] P. Král, I. Thanopulos, and M. Shapiro, Phys. Rev. A 72, 020303 (2005).

[17] Y. Li and C. Bruder, Phys. Rev. A 77, 015403 (2008).

[18] D. V. Kosachiov, B. G. Matisov, and Y. V. Rozhdestvensky, J. Phys. B 25, 2473 (1992).

[19] M. Fleischhauer, R. Unanyan, B. W. Shore, and K. Bergmann, Phys. Rev. A 59, 3751 (1999).

[20] B. Jungnitsch and J. Evers, Phys. Rev. A 78, 043817 (2008).

[21] Y. H. Ma, Q. X. Mu, and L. Zhou, Int. J. Theor. Phys. 46, 3242 (2007).

[22] Y. Makhlin, G. Schon, and A. Shnirman, Rev. Mod. Phys. 73, 357 (2001).

[23] A. Blais, R.-S. Huang, A. Wallraff, S. M. Girvin, and R. J. Schoelkopf, Phys. Rev. A 69, 062320 (2004).

[24] A. Wallraff et al., Nature (London) 431, 162 (2004).

[25] D. I. Schuster et al., Nature (London) 445, 515 (2007).

[26] P. Rabl, D. DeMille, J. M. Doyle, M. D. Lukin, R. J. Schoelkopf, and P. Zoller, Phys. Rev. Lett. 97, 033003 (2006).

[27] M. Kitagawa and M. Ueda, Phys. Rev. A 47, 5138 (1993).

[28] A. S. Rensen, L. M. Duan, I. Cirac, and P. Zoller, Nature (London) 409, 63 (2001).

[29] W. Dür, G. Vidal, and J. I. Cirac, Phys. Rev. A 62, 062314 (2000).

[30] H. R. Zhang, Y. B. Gao, Z. R. Gong, and C. P. Sun, Phys. Rev. A 80, 062308 (2009).

[31] L. M. Duan, J. I. Cirac, and P. Zoller, Phys. Rev. A 66, 023818 (2002).

[32] C. Mewes and M. Fleischhauer, Phys. Rev. A 72, 022327 (2005).

[33] C. P. Sun, Y. Li, and X. F. Liu, Phys. Rev. Lett. 91, 147903 (2003).

[34] J. Hubbard, Proc. R. Soc. London A 277, 237 (1964).

[35] N. N. Bogoliubov, J. Phys. (USSR) 11, 23 (1947).

[36] H. Frochlich, Phys. Rev. 79, 845 (1950).

[37] J. Schrieffer and P. Wolff, Phys. Rev. 149, 491 (1966).

[38] S. Nakajima, Adv. Phys. 4, 463 (1953).

[39] A. D. Greentree, C. Tahan, J. H. Cole, and L. C. L. Hollenberg, Nat. Phys. 2, 856 (2006).
[40] C. D. Ogden, E. K. Irish, and M. S. Kim, Phys. Rev. A 78, 063805 (2008).

[41] S. Tan, J. Opt. B 1, 424 (1999).

[42] S. Rebić, S. M. Tan, A. S. Parkins, and D. F. Walls, J. Opt. B 1, 490 (1999).

[43] M. Hillery and M. S. Zubairy, Phys. Rev. A 74, 032333 (2006).

[44] M. J. Werner and A. Imamoğlu, Phys. Rev. A 61, 011801 (1999).

[45] A. Imamoğlu, H. Schmidt, G. Woods, and M. Deutsch, Phys. Rev. Lett. 79, 1467 (1997).

[46] Y. Li, Y. Castin, and A. Sinatra, Phys. Rev. Lett. 100, 210401 (2008).

[47] M. Scully and M. S. Zubairy, Quantum Optics (Cambridge University Press, Cambridge, UK, 1997).

[48] S. Rebic and J. Twamley, and G. J. Milburn, Phys. Rev. Lett. 103, 150503 (2009).

[49] H. R. Gray, R. Whitley, and J. C. R. Stroud, Opt. Lett. 3, 218 (1978).

[50] D. M. Greenberger, M. Horne, and A. Zeilinger, in Bells Theorem, Quantum Theory, and Conceptions of the Universe, edited by M. Kafatos (Dordrecht, Kluwer, 1989), p. 69.

[51] D. Bouwmeester, J.-W. Pan, M. Daniell, H. Weinfurter, and A. Zeilinger, Phys. Rev. Lett. 82, 1345 (1999).

[52] B. Fortescue and H.-K. Lo, Phys. Rev. Lett. 98, 260501 (2007).

[53] J. Majer et al., Nature (London) 449, 443 (2007).

[54] J. Koch, T. M. Yu, J. Gambetta, A. A. Houck, D. I. Schuster, J. Majer, A. Blais, M. H. Devoret, S. M. Girvin, and R. J. Schoelkopf, Phys. Rev. A 76, 042319 (2007).

[55] J. A. Schreier et al., Phys. Rev. B 77, 180502 (2008).

[56] A. Blais, R. S. Huang, A. Wallraff, S. M. Girvin, and R. J. Schoelkopf, Phys. Rev. A 69, 062320 (2004).

[57] A. Lenef and S. C. Rand, Phys. Rev. B 53, 13441 (1996).

[58] A. Lenef, S. W. Brown, D. A. Redman, S. C. Rand, J. Shigley, and E. Fritsch, Phys. Rev. B 53, 13427 (1996).

[59] C. Santori et al., Opt. Express 14, 7986 (2006).

[60] P. R. Hemmer, A. V. Turukhin, M. S. Shahriar, and J. A. Musser, Opt. Lett. 26, 361 (2001).

[61] P. Tamarat et al., New J. Phys. 10, 045004 (2008).

[62] F. Jelezko et al., Appl. Phys. Lett. 81, 2160 (2002).

[63] G. González and M. N. Leuenberger, Phys. Rev. B 80, 201201(R) (2009).

[64] J. R. Morris and B. W. Shore, Phys. Rev. A 27, 906 (1983).

[65] A. A. Rangelov, N. V. Vitanov, and B. W. Shore, Phys. Rev. A 74, 053402 (2006).

[66] F. E. Zimmer, J. Otterbach, R. G. Unanyan, B. W. Shore, and M. Fleischhauer, Phys. Rev. A 77, 063823 (2008).

[67] A. Blais, J. Gambetta, A. Wallraff, D. I. Schuster, S. M. Girvin, M. H. Devoret, and R. J. Schoelkopf, Phys. Rev. A 75, 032329 (2007).

[68] P. Tamarat et al., Phys. Rev. Lett. 97, 083002 (2006).

[69] F. Bloch, Phys. Rev. 05, 1206 (1957).

[70] A. G. Redfield, IBM J. Res. Dev. 1, 19 (1957).

[71] I. Rau, G. Johansson, and A. Shnirman, Phys. Rev. B 70, 054521 (2004). 